\title{
MPL - a program for computations with iterated integrals on moduli spaces of curves of genus zero
}

\author{
Christian Bogner \\ Institut für Physik, Humboldt-Universität zu Berlin, \\ D - 10099 Berlin, Germany
}

\begin{abstract}
We introduce the computer program MPL for computations with homotopy invariant iterated integrals on moduli spaces $\mathcal{M}_{0, n}$ of curves of genus 0 with $n$ ordered marked points. The program is an implementation of the algorithms presented in [13], based on Maple. It includes the symbol map and procedures for the analytic computation of period integrals on $\mathcal{M}_{0, n}$. It supports the automated computation of a certain class of Feynman integrals.
\end{abstract}




\section{Introduction}

The use of polylogarithms and their generalizations has become standard in the computation of Feynman integrals. A key-advantage of such classes of functions is their double nature as nested sums and iterated integrals, allowing for a choice between computational methods relying on summation techniques and methods based on integral representations.

While classical polylogarithms already appear in one-loop results, the computation of higherloop integrals often requires more general classes of functions. The class of harmonic polylogarithms [58], implemented in [48, 49, 60, 35], serves for many computations, particularly when combined with the method of computing the Feynman integral by solving an associated differential equation [45, 57]. Introducing the dependence on an additional parameter, harmonic polylogarithms were extended 1 in [34, 36]. If one continues along this line of extensions to an arbitrary number of parameters, one arrives at a class of iterated integrals known as hyperlogarithms, considered already in [56] and extensively discussed in [46, 47]. This class of iterated integrals can be used to represent the multiple polylogarithms defined in [37]. Their numerical evaluation was implemented in [61]. An overview of properties of hyperlogarithms and their recent applications in perturbative quantum field theory can be obtained from [53, 32] and references therein.

When writing the Feynman integral in terms of Feynman parameters, it is often possible to successively integrate out these parameters, building up the result as an iterated integral of an appropriate class. By use of hyperlogarithms, this approach was systematized in [18]. Finite integrals can be computed in this way, if certain polynomials in the integrand satisfy the criterion of linear reducibility as defined in [18] and later refined in [19]. This approach for the computation of Feynman integrals in terms of hyperlogarithms was fully implemented in the program HyperInt [54].

Much of the recent progress on the mathematical understanding of Feynman integral results arose from the idea to relate Feynman integrals to period integrals, studied in algebraic geometry. In [9] this correspondence was made explicit by exhibiting a Feynman integral whose result is the period of a motive associated to the first Symanzik polynomial of the Feynman graph. An extensive exploration of periods arising from $\phi^{4}$-theory was conducted in [59]. Furthermore, in a very general context, the coefficients of the Laurent series of dimensionally regularized Feynman integrals are period integrals [15] in the sense of [44]. Recent results at high loop-order suggest, that even though not all periods arising from Feynman graphs are contained in the set of multiple zeta values [25, 23], the 'Feynman periods' seem to constitute a very particular subset of periods with special properties [55].

Multiple zeta values do not only appear in Feynman integral computations but also as periods of

\footnotetext{
${ }^{1}$ Further extensions of harmonic polylogarithms include functions of [1, 8].
} 
moduli spaces of curves of genus zero. In [17] it was proven, that all periods of moduli spaces $\mathcal{M}_{0, n}$ of curves of genus zero with $n \geq 3$ ordered, marked points are multiple zeta values, as previously conjectured in [39]. The proof uses the class of homotopy invariant iterated integrals on these spaces and includes the statement, that this class is closed under taking primitives. The program MPL, introduced in the following, is based on this class of iterated integrals.

In this article, we try to limit the discussion of the mathematical background to aspects of direct importance for the use of the program MPL. All further mathematical details may be obtained from [13] and [17]. Our program MPL is an implementation of the algorithms presented in [13], which in turn are based on [17]. The program can be seen as divided into two main parts. The first part is dedicated to computations with iterated integrals on $\mathcal{M}_{0, n}$, using the framework of so-called cubical coordinates and the corresponding differential 1-forms. Apart from basic operations, such as the shuffle product and the co-product of de-concatenation, the program provides the construction of a basis for the vector space of these iterated integrals by use of the so-called symbol map. It furthermore includes procedures for the differentiation and the derivation of primitives and exact limits at certain points. In particular, it allows for the automated, analytical computation of a class of definite integrals on $\mathfrak{M}_{0, n}$. This class of integrals appears in many different contexts, some of which were already pointed out in [13].

The second part of the program is dedicated to the automated computation of a certain class of Feynman integrals by the mentioned approach of iteratively integrating out Feynman parameters. Here our strategy is the following: For each Feynman parameter, we map the integrand to differential 1-forms in cubical coordinates by an appropriate change of variables. This reduces the problem to an integral on $\mathcal{M}_{0, n}$ of the mentioned class, which we compute by the methods of the first part of the program. Then we map the result of this integration back to an integral only in Feynman parameters and repeat these steps for the remaining parameters. The program constructs and applies such changes of variables, regarding the normalization conditions of both representations at a tangential basepoint.

The class of hyperlogarithms and the class of iterated integrals on $\mathcal{M}_{0, n}$ are equivalently general, in the sense that both can be used to express multiple polylogarithms and each other respectively. In the context of the computation of Feynman integrals, each of these classes of functions comes with its own advantages and drawbacks. The differential 1-forms used to set-up the hyperlogarithms can be defined by direct use of the polynomials, defining the singularities of the given integrand in Feynman parameters. In this way, the integration problem is easily formulated in terms of well adapted iterated integrals. This advantage naturally comes with the inconvenience, that in principle, for each new Feynman integral one works with a new set of iterated integrals.

Using iterated integrals on $\mathcal{M}_{0, n}$ instead, each integral is expressed in terms of a finite basis of functions up to some weight $w$ and number of variables $m$. So in principle, if we consider $w$ and 
$m$ large enough, we will always work with the same set of iterated integrals. On the other hand, in order to use this convenient framework, the given integrand has to be expressed in terms of the particular differential 1-forms in cubical coordinates by a change of variables. The need for such a change of variables introduces certain restrictions on the integrand, to be made precise below. For cases where these conditions are met, the integration procedure is fully implemented in MPL. In other cases, it still may be possible for the user to map the integrand to cubical coordinates 'by hand' and apply MPL afterwards. Of course, one also has to expect cases of Feynman integrals, where such changes of variables do not exist, including the cases where multiple polylogarithms are not sufficient to express the result.

This article is structured as follows. Subsection 1.1 contains information on how to obtain and start the program. Section 2 introduces the framework of iterated integrals on moduli spaces of curves of genus zero in terms of cubical coordinates. Here we discuss basic operations, the symbol map and the construction of the vectorspace of these integrals. In section 3 we discuss the computation of period integrals on the moduli spaces, introducing procedures for taking primitives and certain limits. Section 4 adresses the problem of computing Feynman integrals. Here we specify the conditions under which MPL can be applied and introduce procedures to check these conditions and to compute the integral by iterative integration over Feynman parameters. Section 5 contains our conclusions. In appendix A we give a detailed example of a Feynman integral computation with MPL and in appendix B we give a very basic introduction to moduli spaces of curves of genus zero.

\subsection{How to start the program}

The latest version of MPL is available from the webpage

http://cbogner.com/software/mpl/

The entire program is obtained in one txt-file MPLn_m.txt, where the integers $n$ and $m$ indicate the number of the version. For example, the file of MPL version 1.0 is called MPL1_0.txt. After saving the file in the same directory with the Maple worksheet, the program is started with

$$
\text { >read ("MPL1_0.txt") : }
$$

in the worksheet. While the most important procedures of the program are introduced in this article, further technical details and examples are provided in a user manual, which is also available from the above webpage. MPL was written and tested with Maple 16.

For many applications, it is convenient to let all appearing multiple zeta values be expressed in terms of an irreducible basis automatically. We have used MPL with the file mzv-1-12.txt provided by [7], which serves for this purpose for multiple zeta values up to weight 12 . In the Maple worksheet, it is started simply by 
>read ("mzv-1-12.txt") :

\section{Computing with iterated integrals on moduli spaces of curves of genus zero}

In this section, we define the differential 1-forms and iterated integrals which our implementation is based on. We introduce procedures for basic operations, the symbol and unshuffle maps and a construction of the vectorspace of these iterated integrals.

\subsection{Iterated integrals}

In MPL, every iterated integral is represented by an ordered sequence of differential 1-forms. In order to motivate this notation, let us briefly recall the concept of iterated integrals. For a general introduction to the terminology we recommend [20].

We consider smooth differential 1-forms $\omega_{1}, \ldots, \omega_{k}$ on a smooth, complex manifold $M$ and a smooth path $\gamma:[0,1] \rightarrow M$. An iterated integral along $\gamma$ is defined by

$$
\int_{\gamma} \omega_{1} \ldots \omega_{k}=\int_{0 \leq t_{1} \leq \ldots \leq t_{k} \leq 1} \gamma^{\star}\left(\omega_{k}\right)\left(t_{1}\right) \ldots \gamma^{\star}\left(\omega_{1}\right)\left(t_{k}\right)
$$

where $\gamma^{\star}\left(\omega_{i}\right)\left(t_{j}\right)$ denotes the pull-back of $\omega_{i}$ along $\gamma$, evaluated at $t_{j}$ for $i, j=1, \ldots, k$. We consider the corresponding ordered sequence of 1 -forms $\omega_{1} \otimes \omega_{2} \otimes \ldots \otimes \omega_{k}$ which we write in the so-called bar notation $\left[\omega_{1}\left|\omega_{2}\right| \ldots \mid \omega_{k}\right]$. Note that in our convention, the iterated integration starts with the rightmost 1 -form and proceeds to the left in this sequence. In general we will use the term iterated integral for linear combinations

$$
I=\sum_{J=\left(i_{1}, \ldots, i_{k}\right)} c_{J} \int_{\gamma} \omega_{i_{1}} \ldots \omega_{i_{k}}
$$

of such integrals and we call the corresponding linear combination

$$
\omega=\sum_{J=\left(i_{1}, \ldots, i_{k}\right)} c_{J}\left[\omega_{i_{1}}|\ldots| \omega_{i_{k}}\right]
$$

of sequences the word of $I$.

A famous theorem in [27] implies, that such an iterated integral $I$ is homotopy invariant, if and 
only if its word $\omega$ satisfies the so-called integrability condition

$$
\sum_{J=\left(i_{1}, \ldots, i_{k}\right)} c_{J}\left(\left[\omega_{i_{1}}|\ldots| \omega_{i_{j}} \wedge \omega_{i_{j+1}}|\ldots| \omega_{i_{k}}\right]\right)=0 \text { for all } 1 \leq j \leq k-1
$$

A word $\omega$ satisfying this condition is called integrable. A homotopy invariant iterated integral $I$ depends on the homotopy equivalence class of the path $\gamma$. Let $m$ be the dimension of the manifold $M$ and let us write one of the end-points as $\left(x_{1}, x_{2}, \ldots, x_{m}\right)$ in some coordinates. By convention, we always choose the origin $(0, \ldots, 0)$ as the other end-point of $\gamma$. As we will use differential 1forms with at most logarithmic poles below, one can show that all our iterated integrals admit an expansion of the type

$$
\sum_{J=\left(i_{1}, \ldots, i_{m}\right)} f_{J}\left(x_{1}, \ldots, x_{m}\right) \ln \left(x_{1}\right)^{i_{1}} \ldots \ln \left(x_{m}\right)^{i_{m}}
$$

where the functions $f_{J}$ are analytic (thus in particular convergent) at the origin. This expansion is used to regularize and to normalize the iterated integrals: With respect to cubical coordinates $x_{1}, x_{2}, \ldots, x_{m}$ introduced in the following subsection, the regularized value of the function at the origin is defined to be the term $f_{(0, \ldots, 0)}(0, \ldots, 0)$, i.e. the term obtained by setting all logarithms equal to zero in the expansion. The normalization condition is

$$
f_{(0, \ldots, 0)}(0, \ldots, 0)=0
$$

Due to these conditions, every homotopy invariant iterated integral $I$ is fully determined as a multivalued function of the variables $x_{1}, x_{2}, \ldots, x_{m}$ by the corresponding $\omega$.

Let us remark that in the physics literature, sometimes the term symbol is used as synonym for what we called integrable word here. In some computations where only the differential behaviour of the corresponding iterated integral is relevant, symbols are conveniently used without further conditions with respect to the end-points of $\gamma$. In this case, symbols only represent the function up to contributions of lower length. However, in this article, the above conditions fix this ambiguity and every integrable word determines the corresponding iterated integral entirely. Therefore we use the word $\omega$ to denote the corresponding iterated integral $I$.

In our program, the bar notation is represented by the command bar (...). For example, a word $[a|b| c]$ is represented by

$$
>\operatorname{bar}(\mathrm{a}, \mathrm{b}, \mathrm{c}):
$$

in MPL and stands for the iterated integral obtained by integrating over the 1 -forms in the brackets from right to the left. Numerical multiples are factored out automatically. For example we have:

$$
>\operatorname{bar}(3 * a, 2 * b)+\operatorname{bar}(0) \text {; }
$$




$$
6 \operatorname{bar}(a, b)
$$

Before specifying the differential 1-forms, let us discuss the multiplication and co-multiplication of iterated integrals. Let $a=\left[a_{1}|\ldots| a_{k}\right], b=\left[b_{1}|\ldots| b_{m}\right]$ be two words of some differential 1-forms. By $a \sqcup b$ we denote the concatenation:

$$
a \sqcup b=\left[a_{1}|\ldots| a_{k}\left|b_{1}\right| \ldots \mid b_{m}\right]
$$

The shuffle product $a$ w $b$ is defined by

$$
a \amalg b=\left[a_{1}\right] \sqcup\left(\left[a_{2}|\ldots| a_{k}\right] \amalg b\right)+\left[b_{1}\right] \sqcup\left(a \amalg\left[b_{2}|\ldots| b_{m}\right]\right) .
$$

If $\omega_{1}$ and $\omega_{2}$ are the words of iterated integrals $I_{1}$ and $I_{2}$ respectively, then $\omega_{1} \omega \omega_{2}$ is the word of the product $I_{1} \cdot I_{2}$. In MPL, the shuffle product is implemented in the procedure MPLShuffleProduct $(a, b)$ where the two arguments are the words (in bar notation) to be multiplied with each other. For example:

$$
\begin{aligned}
& >\text { MPLShuffleProduct (bar }(\mathrm{u}, \mathrm{v}), \operatorname{bar}(\mathrm{x}, \mathrm{y})+7 * \operatorname{bar}(\mathrm{z})) ; \\
& 7 \operatorname{bar}(u, v, z)+7 \operatorname{bar}(u, z, v)+7 \operatorname{bar}(z, u, v)+\operatorname{bar}(u, v, x, y)+\operatorname{bar}(u, x, v, y)+\operatorname{bar}(u, x, y, v)+ \\
& \operatorname{bar}(x, u, v, y)+\operatorname{bar}(x, u, y, v)+\operatorname{bar}(x, y, u, v)
\end{aligned}
$$

The de-concatenation co-product $\Delta$, defined by

$$
\Delta\left[a_{1}\left|a_{2}\right| \ldots \mid a_{k}\right]=1 \otimes\left[a_{1}\left|a_{2}\right| \ldots \mid a_{k}\right]+\left[a_{1}\right] \otimes\left[a_{2}|\ldots| a_{k}\right]+\ldots+\left[a_{1}|\ldots| a_{k}\right] \otimes 1
$$

can be computed with the procedure MPLCoproduct (...). Note that MPL returns explicit tensorproducts of words by use of tens (...).

\subsection{Differential 1-forms in cubical coordinates}

The program MPL is based on the class of homotopy invariant iterated integrals on moduli spaces $\mathcal{M}_{0, n}$ of curves with $n \geq 3$ ordered, marked points. These spaces and this class of functions are extensively studied in [17]. In appendix B, we give a very basic introduction to the spaces $\mathcal{M}_{0, n}$. However, without giving a full account on the underlying geometry here, the mentioned class of iterated integrals can be specified as follows. For $m=n-3$ let us consider the set $\Omega_{m}$ of differential 1 -forms, defined by

$$
\Omega_{m}=\left\{\frac{d x_{1}}{x_{1}}, \ldots, \frac{d x_{m}}{x_{m}}, \frac{d\left(\prod_{a \leq i \leq b} x_{i}\right)}{\prod_{a \leq i \leq b} x_{i}-1} \text { for } 1 \leq a \leq b \leq m\right\} .
$$


Let $\mathcal{A}_{m}$ be the $\mathbb{Q}$-vectorspace spanned by $\Omega_{m}$. By the class of iterated integrals on $\mathcal{M}_{0, n}$ we refer to the $\mathbb{Q}$-vectorspace $V\left(\Omega_{m}\right)$ of homotopy invariant iterated integrals of differential 1-forms in $\mathcal{A}_{m}$, regularized and normalized by the conditions introduced in section 2.1.

The variables $x_{1}, \ldots, x_{m}$ in the above 1 -forms are called cubical coordinates in this context. We will sometimes refer to $x_{m}$ as the last of the cubical coordinates. In the Maple worksheet, the set of cubical coordinates has to be declared before many of the computations below. With the command MPLCoordinates (letter, $m$ ) one declares $m$ cubical coordinates, where the first argument is used to construct the variable names. For example after the command

$>$ MPLCoordinates $(\mathrm{y}, 3)$ :

we can compute with y [1], y [2], y [3].

Following [13], let us furthermore introduce the auxiliary sets of differential 1-forms

$$
\begin{aligned}
& \bar{\Omega}_{m}^{F}=\left\{\frac{d x_{m}}{x_{m}}, \frac{\left(\prod_{a \leq i \leq m-1} x_{i}\right) d x_{m}}{\prod_{a \leq i \leq m} x_{i}-1} \text { for } 1 \leq a \leq m\right\}, \\
& \Omega_{m}^{F}=\left\{\frac{d x_{m}}{x_{m}}, \frac{d\left(\prod_{a \leq i \leq m} x_{i}\right)}{\prod_{a \leq i \leq m} x_{i}-1} \text { for } 1 \leq a \leq m\right\},
\end{aligned}
$$

noting that $\Omega_{m}=\Omega_{m}^{F} \cup \Omega_{m-1}$. We define $\overline{\mathcal{A}}_{m}^{F}, \mathcal{A}_{m}^{F}$ to be the $\mathbb{Q}$-vectorspaces of differential 1-forms, spanned by the bases $\bar{\Omega}_{m}^{F}, \Omega_{m}^{F}$ respectively. An isomorphism between these spaces is given by

$$
\begin{aligned}
\lambda_{m}: \overline{\mathcal{A}}_{m}^{F} & \rightarrow \mathcal{A}_{m}^{F}, \\
\frac{d x_{m}}{x_{m}} & \mapsto \frac{d x_{m}}{x_{m}}, \\
\frac{\left(\prod_{a \leq i \leq m-1} x_{i}\right) d x_{m}}{\prod_{a \leq i \leq m} x_{i}-1} & \mapsto \frac{d\left(\prod_{a \leq i \leq m} x_{i}\right)}{\prod_{a \leq i \leq m} x_{i}-1} .
\end{aligned}
$$

The $\mathbb{Q}$-vectorspace $V\left(\bar{\Omega}_{m}^{F}\right)$ of iterated integrals with all 1-forms in $\overline{\mathcal{A}}_{m}^{F}$ plays an auxiliary role in some computations. While the iterated integrals in $V\left(\Omega_{m}\right)$ are functions of $m$ variables on $\mathcal{M}_{0, n}$, $V\left(\bar{\Omega}_{m}^{F}\right)$ is a space of hyperlogarithms, being functions of the one variable $x_{m}$ on a fiber over $\mathcal{M}_{0, n-1}$, with the $x_{1}, \ldots, x_{m-1}$ considered fixed. Note that every word in $\overline{\mathcal{A}}_{m}^{F}$ is integrable by construction of $\bar{\Omega}_{m}^{F}$, but words in $\mathcal{A}_{m}$ can fail the integrability condition. An explicit construction of all integrable words in $\mathcal{A}_{m}$, implying the construction of $V\left(\Omega_{m}\right)$, is discussed in section 2.4,

Let us mention that the above differential 1-forms satisfy quadratic relations due to Arnold [5], of the form

$$
\omega_{i} \wedge \omega_{j}=\sum_{k} \alpha_{k} \wedge \omega_{k}
$$

with $\omega_{i} \in \Omega_{m}^{F} \alpha_{i} \in \Omega_{m-1}$. These Arnold equations are explicitely given in [13] and are of inter- 
nal importance for our algorithms. By the command MPLArnoldEquation (...), they are also available for the user. We refer to the manual for details.

\subsection{Differentiation}

There are several notions of differentiation on $V\left(\Omega_{m}\right)$ and $V\left(\bar{\Omega}_{m}^{F}\right)$. For both spaces, the differentiation $d$ with respect to the end-point of the path is simply the truncation of the leftmost 1-form:

$$
\begin{aligned}
d: V\left(\Omega_{m}\right) & \rightarrow \mathcal{A}_{m} \otimes V\left(\Omega_{m}\right) \\
\sum_{J=\left(i_{1}, \ldots, i_{k}\right)} c_{J}\left[\omega_{i_{1}}|\ldots| \omega_{i_{k}}\right] & \mapsto \sum_{J=\left(i_{1}, \ldots, i_{k}\right)} c_{J} \omega_{i_{1}} \otimes\left[\omega_{i_{2}}|\ldots| \omega_{i_{k}}\right] .
\end{aligned}
$$

This operation is well-known from the literature on hyperlogarithms. It is implemented in the procedure MPLd (...).

A connection is a linear map

$$
\nabla: V\left(\bar{\Omega}_{m}^{F}\right) \rightarrow \mathcal{A}_{m-1} \otimes V\left(\bar{\Omega}_{m}^{F}\right)
$$

satisfying the Leibniz rule. In our framework, it can be constructed [13] by firstly applying the map $\lambda_{m}$ to a word in $\overline{\mathcal{A}}_{m}^{F}$, then applying an operator $D$ defined by

$$
D\left[\omega_{1}|\ldots| \omega_{k}\right]=(-1)^{k} \sum_{i=1}^{k-1}\left[\omega_{1}|\ldots| \omega_{i} \wedge \omega_{i+1} \mid \omega_{k}\right]
$$

then expressing the wedge products on the right-hand in the form $\sum_{k} \alpha_{k} \wedge \omega_{k}$ with $\omega_{i} \in \Omega_{m}^{F} \alpha_{i} \in$ $\Omega_{m-1}$ by use of the Arnold equations eq. 4 and finally pulling out all $\alpha_{i}$ to the left. With the help of the connection $\nabla$, the total connection

$$
\nabla_{T}: V\left(\bar{\Omega}_{m}^{F}\right) \rightarrow \mathcal{A}_{m} \otimes V\left(\bar{\Omega}_{m}^{F}\right)
$$

is defined by

$$
\nabla_{T}=d-\nabla
$$

It is implemented in the procedure MPLTotalConnection $(. .$.$) . We refer to the manual for exam-$ ples. 


\subsection{The symbol map and the construction of $V\left(\Omega_{m}\right)$}

As not every word in $\mathcal{A}_{m}$ is integrable, the construction of the vectorspace $V\left(\Omega_{m}\right)$ is not trivial. For example let $m=3$ and consider the words

$$
\omega_{1}=\left[\frac{d x_{3}}{x_{3}}+\frac{d x_{2}}{x_{2}} \mid \frac{d\left(x_{2} x_{3}\right)}{x_{2} x_{3}-1}\right] \text { and } \omega_{2}=\left[\frac{d x_{3}}{x_{3}} \mid \frac{d\left(x_{2} x_{3}\right)}{x_{2} x_{3}-1}\right] .
$$

The word $\omega_{1}$ is integrable, but $\omega_{2}$ is not. MPL does not prohibit the use of non-integrable words, and many operations can be applied to $\omega_{2}$ as well as to $\omega_{1}$. However, $\omega_{2}$ does not represent an iterated integral in our framework. In many applications, the user does not need to worry about this point. If MPL is used for the computation of an integral, the program returns the result in terms of iterated integrals which are homotopy invariant by construction. However, for some applications, it may be useful to have an explicit basis for $V\left(\Omega_{m}\right)$ up to a certain weight at hand 2 .

The construction of this basis is facilitated by the so-called symbol map

$$
\Psi: V\left(\bar{\Omega}_{m}^{F}\right) \rightarrow V\left(\Omega_{m}\right)
$$

It is the unique linear map satisfying

$$
(i d \otimes \Psi) \circ \nabla_{T}=d \circ \Psi
$$

This map was explicitely constructed in [12, 13] and is related to the constructions of the symbol in [33, 38, 40]. It is implemented in MPLSymbolMap ( . . ) .

\section{Example:}

We apply the symbol map $\Psi$ to the hyperlogarithm $\left[\frac{d x_{3}}{x_{3}} \mid \frac{x_{2} d\left(x_{3}\right)}{1-x_{2} x_{3}}\right] \in V\left(\bar{\Omega}_{m}^{F}\right)$ :

$$
\begin{aligned}
& >\text { MPLCoordinates }(\mathrm{x}, 3): \\
& >\text { MPLSymbolMap }(\operatorname{bar}(\mathrm{d}(\mathrm{x}[3]) /(\mathrm{x}[3]), \mathrm{x}[2] * \mathrm{~d}(\mathrm{x}[3]) /(1-\mathrm{x}[2] * \mathrm{x}[3]))) ; \\
& \operatorname{bar}(d(x[3]) / x[3],(x[3] * d(x[2])+x[2] * d(x[3])) /(1-x[2] * x[3]))+\operatorname{bar}(d(x[2]) / x[2],(x[3] * \\
& d(x[2])+x[2] * d(x[3])) /(1-x[2] * x[3]))
\end{aligned}
$$

Slightly simplifying this output, we obtain

$$
\Psi\left(\left[\frac{d x_{3}}{x_{3}} \mid \frac{x_{2} d\left(x_{3}\right)}{1-x_{2} x_{3}}\right]\right)=\left[\frac{d x_{3}}{x_{3}}+\frac{d x_{2}}{x_{2}} \mid \frac{d\left(x_{2} x_{3}\right)}{1-x_{2} x_{3}}\right] \in V\left(\Omega_{3}\right) .
$$

\footnotetext{
${ }^{2}$ The construction of certain subspaces of integrable words played an important role e.g. in the recent computations [30, 29, 31].
} 
Let $\bar{B}_{m, w}$ be the set of iterated integrals with words $\omega=\left[\omega_{1}|\ldots| \omega_{k}\right]$ with $k \leq w$ and with all 1forms in $\bar{\Omega}_{m}^{F}$. This set $\bar{B}_{m, w}$ is a basis for the subspace $V_{w}\left(\bar{\Omega}_{m}^{F}\right) \subset V\left(\bar{\Omega}_{m}^{F}\right)$ of iterated integrals whose words in $\overline{\mathcal{A}}_{m}^{F}$ are of length $k \leq w$. Let $B_{m, w}$ be the desired basis of the subspace $V_{w}\left(\Omega_{m}\right) \subset V\left(\Omega_{m}\right)$ of words of length $k \leq w$. A theorem of [17] states the existence of an isomorphism of algebras

$$
V\left(\Omega_{m}\right) \cong V\left(\Omega_{m-1}\right) \otimes V\left(\bar{\Omega}_{m}^{F}\right)
$$

As a consequence, $B_{m, w}$ is the set of all iterated integrals whose words $\omega$ are of length $k \leq w$ and are obtained as products $\omega=\eta_{\amalg} \Psi(\xi)$ with $\eta \in B_{m-1, w}$ and $\xi \in \bar{B}_{m, w}$. We can formulate this construction by the map

$$
\mu(i d \otimes \Psi): V\left(\Omega_{m-1}\right) \otimes V\left(\bar{\Omega}_{m}^{F}\right) \rightarrow V\left(\Omega_{m}\right)
$$

where $\mu$ denotes multiplication. This provides a recursive construction of the basis $B_{m, w}$. The procedure MPLBasis (letter, $\mathrm{m}, \mathrm{w}$ ) returns $B_{m, w}$ for words up to weight w in variables named by the first argument.

\section{Example:}

$$
\begin{aligned}
& >\operatorname{MPLBasis}(\mathrm{y}, 2,2) \text {; } \\
& {\left[\left[\operatorname{bar}\left(\frac{d\left(y_{2}\right)}{y_{2}}\right), \operatorname{bar}\left(\frac{d\left(y_{2}\right)}{1-y_{2}}\right), \operatorname{bar}\left(\frac{y_{2} d\left(y_{1}\right)+y_{1} d\left(y_{2}\right)}{1-y_{1} y_{2}}\right), \operatorname{bar}\left(\frac{d\left(y_{1}\right)}{y_{1}}\right), \operatorname{bar}\left(\frac{d\left(y_{1}\right)}{1-y_{1}}\right)\right]\right.} \\
& {\left[\operatorname{bar}\left(\frac{d\left(y_{2}\right)}{y_{2}}, \frac{d\left(y_{2}\right)}{y_{2}}\right), \operatorname{bar}\left(\frac{d\left(y_{2}\right)}{y_{2}}, \frac{d\left(y_{2}\right)}{1-y_{2}}\right), \operatorname{bar}\left(\frac{d\left(y_{2}\right)}{1-y_{2}}, \frac{d\left(y_{2}\right)}{y_{2}}\right),\right.} \\
& \operatorname{bar}\left(\frac{d\left(y_{2}\right)}{y_{2}}, \frac{y_{2} d\left(y_{1}\right)+y_{1} d\left(y_{2}\right)}{1-y_{1} y_{2}}\right)+\operatorname{bar}\left(\frac{d\left(y_{1}\right)}{y_{1}}, \frac{y_{2} d\left(y_{1}\right)+y_{1} d\left(y_{2}\right)}{1-y_{1} y_{2}}\right) \text {, } \\
& \operatorname{bar}\left(\frac{y_{2} d\left(y_{1}\right)+y_{1} d\left(y_{2}\right)}{1-y_{1} y_{2}}, \frac{d\left(y_{2}\right)}{y_{2}}\right)-\operatorname{bar}\left(\frac{d\left(y_{1}\right)}{y_{1}}, \frac{y_{2} d\left(y_{1}\right)+y_{1} d\left(y_{2}\right)}{1-y_{1} y_{2}}\right), \operatorname{bar}\left(\frac{d\left(y_{2}\right)}{1-y_{2}}, \frac{d\left(y_{2}\right)}{1-y_{2}}\right) \text {, } \\
& \operatorname{bar}\left(\frac{d\left(y_{2}\right)}{1-y_{2}}, \frac{y_{2} d\left(y_{1}\right)+y_{1} d\left(y_{2}\right)}{1-y_{1} y_{2}}\right)-\operatorname{bar}\left(\frac{d\left(y_{1}\right)}{1-y_{1}}, \frac{y_{2} d\left(y_{1}\right)+y_{1} d\left(y_{2}\right)}{1-y_{1} y_{2}}\right)+\operatorname{bar}\left(\frac{d\left(y_{1}\right)}{1-y_{1}}, \frac{d\left(y_{2}\right)}{1-y_{2}}\right) \\
& -\operatorname{bar}\left(\frac{d\left(y_{1}\right)}{y_{1}}, \frac{y_{2} d\left(y_{1}\right)+y_{1} d\left(y_{2}\right)}{1-y_{1} y_{2}}\right), \operatorname{bar}\left(\frac{y_{2} d\left(y_{1}\right)+y_{1} d\left(y_{2}\right)}{1-y_{1} y_{2}}, \frac{d\left(y_{2}\right)}{1-y_{2}}\right) \\
& +\operatorname{bar}\left(\frac{d\left(y_{1}\right)}{1-y_{1}}, \frac{y_{2} d\left(y_{1}\right)+y_{1} d\left(y_{2}\right)}{1-y_{1} y_{2}}\right)-\operatorname{bar}\left(\frac{d\left(y_{1}\right)}{1-y_{1}}, \frac{d\left(y_{2}\right)}{1-y_{2}}\right) \\
& +\operatorname{bar}\left(\frac{d\left(y_{1}\right)}{y_{1}}, \frac{y_{2} d\left(y_{1}\right)+y_{1} d\left(y_{2}\right)}{1-y_{1} y_{2}}\right), \operatorname{bar}\left(\frac{y_{2} d\left(y_{1}\right)+y_{1} d\left(y_{2}\right)}{1-y_{1} y_{2}}, \frac{y_{2} d\left(y_{1}\right)+y_{1} d\left(y_{2}\right)}{1-y_{1} y_{2}}\right) \text {, }
\end{aligned}
$$




$$
\begin{gathered}
\operatorname{bar}\left(\frac{d\left(y_{1}\right)}{y_{1}}, \frac{d\left(y_{2}\right)}{y_{2}}\right)+\operatorname{bar}\left(\frac{d\left(y_{2}\right)}{y_{2}}, \frac{d\left(y_{1}\right)}{y_{1}}\right), \operatorname{bar}\left(\frac{d\left(y_{1}\right)}{y_{1}}, \frac{d\left(y_{2}\right)}{1-y_{2}}\right) \\
+\operatorname{bar}\left(\frac{d\left(y_{2}\right)}{1-y_{2}}, \frac{d\left(y_{1}\right)}{y_{1}}\right), \operatorname{bar}\left(\frac{d\left(y_{1}\right)}{y_{1}}, \frac{y_{2} d\left(y_{1}\right)+y_{1} d\left(y_{2}\right)}{1-y_{1} y_{2}}\right)+\operatorname{bar}\left(\frac{y_{2} d\left(y_{1}\right)+y_{1} d\left(y_{2}\right)}{1-y_{1} y_{2}}, \frac{d\left(y_{1}\right)}{y_{1}}\right), \\
\operatorname{bar}\left(\frac{d\left(y_{1}\right)}{1-y_{1}}, \frac{d\left(y_{2}\right)}{y_{2}}\right)+\operatorname{bar}\left(\frac{d\left(y_{2}\right)}{y_{2}}, \frac{d\left(y_{1}\right)}{1-y_{1}}\right), \operatorname{bar}\left(\frac{d\left(y_{1}\right)}{1-y_{1}}, \frac{d\left(y_{2}\right)}{1-y_{2}}\right)+\operatorname{bar}\left(\frac{d\left(y_{2}\right)}{1-y_{2}}, \frac{d\left(y_{1}\right)}{1-y_{1}}\right), \\
\operatorname{bar}\left(\frac{d\left(y_{1}\right)}{1-y_{1}}, \frac{y_{2} d\left(y_{1}\right)+y_{1} d\left(y_{2}\right)}{1-y_{1} y_{2}}\right)+\operatorname{bar}\left(\frac{y_{2} d\left(y_{1}\right)+y_{1} d\left(y_{2}\right)}{1-y_{1} y_{2}}, \frac{d\left(y_{1}\right)}{1-y_{1}}\right), \operatorname{bar}\left(\frac{d\left(y_{1}\right)}{y_{1}}, \frac{d\left(y_{1}\right)}{y_{1}}\right), \\
\left.\left.\operatorname{bar}\left(\frac{d\left(y_{1}\right)}{y_{1}}, \frac{d\left(y_{1}\right)}{1-y_{1}}\right), \operatorname{bar}\left(\frac{d\left(y_{1}\right)}{1-y_{1}}, \frac{d\left(y_{1}\right)}{y_{1}}\right), \operatorname{bar}\left(\frac{d\left(y_{1}\right)}{1-y_{1}}, \frac{d\left(y_{1}\right)}{1-y_{1}}\right)\right]\right]
\end{gathered}
$$

This result is a basis for iterated integrals in $V\left(\Omega_{2}\right)$ in variables $y_{1}, y_{2}, y_{3}, y_{4}$ up to weight 2 as sequence of two lists $S=\left[S_{1}, S_{2}\right]$. The list $S_{1}$ contains the basis for weight 1 and $S_{2}$ for weight 2 .

\subsection{The unshuffle map and hyperlogarithms}

There is an explicit construction [13] of the inverse of the map of eq. 7]called the unshuffle map:

$$
\Phi: V\left(\Omega_{m}\right) \rightarrow V\left(\Omega_{m-1}\right) \otimes V\left(\bar{\Omega}_{m}^{F}\right)
$$

It is available by the procedure MPLUnshuffle ( $f$, var), which decomposes a function $f$, such that

the right component of tensor-product in eq. 8 is a hyperlogarithm in the variable var. The variable var has to be the last of the declared cubical coordinates.

\section{Example:}

Consider the function

$$
f=\left[2 \frac{d x_{2}}{x_{2}}+\frac{d x_{3}}{x_{3}}\left|\frac{d x_{2}}{x_{2}}\right| \frac{d\left(x_{2} x_{3}\right)}{1-x_{2} x_{3}}\right]+\left[\frac{d x_{2}}{x_{2}}\left|\frac{d x_{3}}{x_{3}}\right| \frac{d\left(x_{2} x_{3}\right)}{1-x_{2} x_{3}}\right]+\left[\frac{d x_{2}}{x_{2}}+\frac{d x_{3}}{x_{3}}\left|\frac{d\left(x_{2} x_{3}\right)}{1-x_{2} x_{3}}\right| \frac{d x_{2}}{x_{2}}\right] \in V\left(\Omega_{3}\right) .
$$

We apply MPLUnshuffle to $f$ with respect to variable $x_{3}$ :

$$
\begin{aligned}
& >\text { MPLCoordinates }(\mathrm{x}, 3) \text { : } \\
& >\text { MPLUnshuffle }(\mathrm{f}, \mathrm{x}[3]) ; \\
& \text { tens }(\operatorname{bar}(d(x[2]) / x[2]), \operatorname{bar}(d(x[3]) / x[3], x[2] d(x[3]) /(1-x[2] x[3])))
\end{aligned}
$$

This output is understood as

$$
\left[\frac{d x_{2}}{x_{2}}\right] \otimes\left[\frac{d x_{3}}{x_{3}} \mid \frac{x_{2} d\left(x_{3}\right)}{1-x_{2} x_{3}}\right]
$$


We see that the left part of this tensor product is a function in $V\left(\Omega_{2}\right)$ (in this simple example, it even belongs to $V\left(\bar{\Omega}_{2}^{F}\right)$ ), while the right part is a hyperlogarithm in $V\left(\bar{\Omega}_{3}^{F}\right)$. Hence, as easily confirmed using eq. [5, the function $f$ can be constructed as

$$
f=\left[\frac{d x_{2}}{x_{2}}\right] \amalg \Psi\left(\left[\frac{d x_{3}}{x_{3}} \mid \frac{x_{2} d\left(x_{3}\right)}{1-x_{2} x_{3}}\right]\right) .
$$

In the same recursive way as the shuffle map $\Psi$ can be used to construct all iterated integrals in $V\left(\Omega_{m}\right)$ from hyperlogarithms in $V\left(\bar{\Omega}_{k}^{F}\right)$ with $k \leq m$, the recursive application of the unshuffle map $\Phi$ to the left part of the tensor product in eq. 8 decomposes every function in $V\left(\Omega_{m}\right)$ as a product of such hyperlogarithms. In this way, one can express all functions of $V\left(\Omega_{m}\right)$ in terms of hyperlogarithms and vice versa.

\section{Integration over cubical coordinates}

In this section, we introduce procedures for the computation of primitives and certain limits of iterated integrals in $V\left(\Omega_{m}\right)$. A further command which combines these computations serves for the analytical computation of certain definite integrals on $\mathcal{M}_{0, n}$. These procedures will also be the backbone of the Feynman integral computations of section 4

\subsection{Primitives}

It is well-known and implied by the definition of hyperlogarithms, that the computation of the primitive of a hyperlogarithm with respect to some differential 1 -form $\omega_{0}$ is simply the left-concatenation of this 1 -form to the corresponding word. In our set-up this is a map

$$
\begin{aligned}
\overline{\mathcal{A}}_{m}^{F} \otimes V\left(\bar{\Omega}_{m}^{F}\right) & \rightarrow V\left(\bar{\Omega}_{m}^{F}\right), \\
\omega_{0} \otimes\left[\omega_{1}|\ldots| \omega_{k}\right] & \mapsto \int \omega_{0} \otimes\left[\omega_{1}|\ldots| \omega_{k}\right]=\left[\omega_{0}\left|\omega_{1}\right| \ldots \mid \omega_{k}\right] .
\end{aligned}
$$

As every word in $\overline{\mathcal{A}}_{m}^{F}$ is integrable, every primitive obtained in this way is trivially homotopy invariant.

However, the computation of a primitive of a function $f \in V\left(\Omega_{m}\right)$ has to be different from simple left-concatenation in general. This can already be understood from the simple fact that there are 1-forms $\omega_{0}$ and integrable words $\omega$ with letters in $\Omega_{m}$, such that the concatenation $\omega_{0} \sqcup \omega$ is not integrable. However, the primitive of a function in $V\left(\Omega_{m}\right)$ with respect to a 1-form in $\overline{\mathcal{A}}_{m}^{F}$ is given by a map 


$$
\begin{aligned}
\overline{\mathcal{A}}_{m}^{F} \otimes V\left(\Omega_{m}\right) & \rightarrow V\left(\Omega_{m}\right) \\
\omega_{0} \otimes\left[\omega_{1}|\ldots| \omega_{k}\right] & \mapsto \int \omega_{0} \otimes\left[\omega_{1}|\ldots| \omega_{k}\right]
\end{aligned}
$$

whose existence is implied by a theorem of [17].

In principle, an explicit computation of primitives could start from a decomposition into hyperlogarithms as in section 2.5, apply simple concatenation as in eq. 9 and then map back to $V\left(\Omega_{m}\right)$ by use of the symbol map. However, a more efficient algorithm, avoiding the decomposition into hyperlogarithms, was presented in [13]. This algorithm is implemented in MPL. For a 1-form a in $\overline{\mathcal{A}}_{m}^{F}$ and a function $\mathrm{f}$ in $V\left(\Omega_{m}\right)$ whose maximal weight is $\mathrm{w}$, the procedure MPLPrimitive (a, $\mathrm{f}$, w) computes the primitive $\int a \otimes f \in V\left(\Omega_{m}\right)$.

\section{Example:}

Let us consider the function

$$
f=\left[\frac{d x_{2}}{x_{2}}+\frac{d x_{3}}{x_{3}} \mid \frac{d\left(x_{2} x_{3}\right)}{1-x_{2} x_{3}}\right]+17\left[\frac{d x_{3}}{1-x_{3}}\right] \in V\left(\Omega_{3}\right)
$$

whose maximal weight is $w=2$, and the 1 -form $a=\frac{d x_{3}}{1-x_{3}} \in \overline{\mathcal{A}}_{3}^{F}$. By use of the commands

$>\operatorname{MPLCoordinates}(\mathrm{x}, 3)$ :

$>\operatorname{MPLPrimitive}(a, f, 2)$;

we obtain

$$
\begin{aligned}
\int a \otimes f= & {\left[\frac{d x_{3}}{1-x_{3}}\left|\frac{d x_{3}}{x_{3}}\right| \frac{d\left(x_{2} x_{3}\right)}{1-x_{2} x_{3}}\right]+\left[\frac{d x_{3}}{1-x_{3}}-\frac{d x_{2}}{x_{2}}\left|\frac{d x_{2}}{x_{2}}\right| \frac{d\left(x_{2} x_{3}\right)}{1-x_{2} x_{3}}\right]+\left[\frac{d x_{2}}{x_{2}}\left|\frac{d x_{3}}{1-x_{3}}\right| \frac{d\left(x_{2} x_{3}\right)}{1-x_{2} x_{3}}\right] } \\
& +\left[\frac{d x_{2}}{x_{2}}\left|\frac{d x_{2}}{1-x_{2}}\right| \frac{d x_{3}}{1-x_{3}}-\frac{d\left(x_{2} x_{3}\right)}{1-x_{2} x_{3}}\right]+17\left[\frac{d x_{3}}{1-x_{3}} \mid \frac{d x_{3}}{1-x_{3}}\right] \in V\left(\Omega_{3}\right) .
\end{aligned}
$$

\subsection{Limits}

MPL can be used to take limits of functions $f \in V\left(\Omega_{m}\right)$ at $x_{k}=u$ where $u \in\{0,1\}$ and where $x_{k}$ is any of the cubical coordinates $x_{1}, \ldots, x_{m}$. Let $Z$ be the $\mathbb{Q}$-vectorspace of multiple zeta values. It was proven in [17] that for every $f \in V\left(\Omega_{m}\right)$ the $\operatorname{limits}_{x_{k} \rightarrow u} f$ are $Z$-linear combinations of functions in $V\left(\Omega_{m-1}\right)$. 
If the limit is computed with respect to the last variable $x_{m}$, the limit is readily expressed in

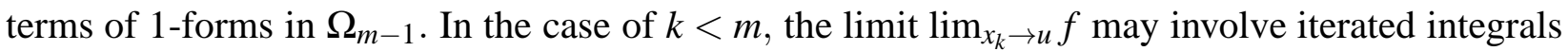
in differential 1 -forms of the type $\frac{d\left(x_{i} x_{i+1} \ldots \hat{x}_{k} \ldots x_{j}\right)}{1-x_{i} x_{i+1} \ldots \hat{x}_{k} \ldots x_{j}}$, where the hat indicates the missing variable $x_{k}$ in the products of consecutive coordinates. Here one has to re-name the coordinates $\left(x_{k+1}, \ldots, x_{m}\right) \mapsto$ $\left(x_{k}, \ldots, x_{m-1}\right)$, such that these 1-forms belong to $\Omega_{m-1}$ and the iterated integrals are recognized to belong to $V\left(\Omega_{m-1}\right)$ by MPL.

The limits are computed by expansion of the given $f \in V\left(\Omega_{m}\right)$ as a series in $x_{k}=u$, and evaluation of the coefficient of $\ln (x-u)^{0}$ (according to the regularization condition of section 2.1). The expansion makes use of the decomposition eq. 6 such that the problem is internally reduced to the computation of regularized limits of functions in $V\left(\Omega_{1}\right)$. The analytical solution to the latter problem is known and leads to elements of $Z$ (see [21]). The computation does not involve any numerical approximation. The command MPLLimit $(\mathrm{f}, \mathrm{x}[\mathrm{k}], \mathrm{u})$ returns the limit of $f \in V\left(\Omega_{m}\right)$ at $x_{k}=u$ with $u \in\{0,1\}$.

\section{Example:}

Consider the function

$$
f=\Psi\left(\left[\frac{d x_{2}}{1-x_{2}} \mid \frac{x_{1} d x_{2}}{1-x_{1} x_{2}}\right]\right)=\left[\frac{d x_{2}}{1-x_{2}}-\frac{d x_{1}}{1-x_{1}}-\frac{d x_{1}}{x_{1}} \mid \frac{d\left(x_{1} x_{2}\right)}{1-x_{1} x_{2}}\right]+\left[\frac{d x_{1}}{1-x_{1}} \mid \frac{d x_{2}}{1-x_{2}}\right] \in V\left(\Omega_{2}\right) .
$$

By use of the commands

$$
\begin{aligned}
& >\operatorname{MPLCoordinates}(\mathrm{x}, 2) \text { : } \\
& >\operatorname{MPLLimit}(\mathrm{f}, \mathrm{x}[2], 1) ;
\end{aligned}
$$

we obtain the limit

$$
\lim _{x_{2} \rightarrow 1} f=-\left[\frac{d x_{1}}{1-x_{1}}+\frac{d x_{1}}{x_{1}} \mid \frac{d x_{1}}{1-x_{1}}\right]=-\frac{1}{2} \ln \left(1-x_{1}\right)^{2}-\operatorname{Li}_{2}\left(x_{1}\right) \in V\left(\Omega_{2}\right)
$$

where $\mathrm{Li}_{2}$ denotes the classical dilogarithm.

In some applications it is necessary to compute several consecutive limits

$$
\lim _{x_{k_{1}} \rightarrow u_{1}} \lim _{x_{2} \rightarrow u_{2}} \ldots \lim _{x_{k_{l}} \rightarrow u_{l}} f
$$

with $\left\{k_{1}, \ldots, k_{l}\right\} \subseteq\{1, \ldots, m\}$ and $u_{i} \in\{0,1\}$ for $i \in\{1, \ldots, l\}$. Such computations are facilitated by the procedure MPLMultipleLimit $(\mathrm{f}, \mathrm{S})$ where the first argument is a function in $V\left(\Omega_{m}\right)$ and the second argument is an ordered list $S=[\ldots]$ of equations $x_{k_{l}}=u_{l}, x_{k_{l-1}}=u_{l-1}, \ldots, x_{k_{1}}=u_{1}$ which define the limits. The order in which the limits are computed is from left to right in this list. 
It is important to note that in such multiple limits, the order matters. For example, for $f$ defined in eq. 10, we have

$$
\begin{aligned}
& \lim _{x_{1} \rightarrow 1} \lim _{x_{2} \rightarrow 1} f=-\zeta(2), \\
& \lim _{x_{2} \rightarrow 1} \lim _{x_{1} \rightarrow 1} f=0 .
\end{aligned}
$$

In both computations, we approach the point $\left(x_{1}, x_{2}\right)=(1,1)$. In the first line, we evaluate $f$ on the boundary $L_{1}=\left\{\left(x_{1}, 1\right) \mid 0 \leq x_{1} \leq 1\right\}$ at first and then approach the point $(1,1)$ along this line. In

the second computation, we move to the boundary $L_{2}=\left\{\left(1, x_{2}\right) \mid 0 \leq x_{2} \leq 1\right\}$ at first and approach the point $(1,1)$ from there. The difference between both results comes from the fact, that the point $(1,1)$ is not contained in the moduli space $\mathcal{M}_{0,5}$ (see appendix $\mathrm{B}$, eq. 25). In order to evaluate functions there, one has to work with a compactification of this space, obtained by blowing up this point to a line, such that the boundary transforms from a square to a pentagon in this case. On this space, one clearly arrives at different points, depending on whether one approaches the additional line from $L_{1}$ or $L_{2}$. We refer to [17] for a detailed, general discussion.

\subsection{Definite integration}

Let us consider cubical coordinates $x_{1}, \ldots, x_{m}$ and convergent integrals of the form

$$
I=\int_{0}^{1} d x_{m} \frac{q}{\prod_{i} p_{i}^{a_{i}}} f
$$

where $f \in V\left(\Omega_{m}\right), q$ is a polynomial in $x_{m}$, the $a_{i} \in \mathbb{N}$ and the $p_{i}$ are elements of the set

$$
\mathcal{P}_{C}=\left\{x_{m}, 1-x_{m}, \ldots, 1-x_{1} \cdots x_{m}\right\}
$$

i.e. in the set of denominators of the differential 1-forms in $\Omega_{m}$. The analytical computation of such integrals with MPL proceeds as follows:

- The integral $I$ is expressed as a linear combination of integrals of the type

$$
I_{i}=\int_{0}^{1} \omega_{i} f_{i}
$$

with $\omega_{i} \in \bar{\Omega}_{m}^{F}, f_{i} \in V\left(\Omega_{m}\right)$. This is achieved by a combination of partial fraction decompositions and partial integrations, iteratively lowering the exponents $a_{i}$. The partial integrations involve the computation of limits of $f$ at $x_{m}=0$ and $x_{m}=1$ as discussed in section 3.2 .

- For each integral $I_{i}$ the primitive $\tilde{I}_{i}=\int \omega_{i} \otimes f_{i}$ is computed as in section 3.1 
- The limits $\lim _{x_{m} \rightarrow 1} \tilde{I}_{i}-\lim _{x_{m} \rightarrow 0} \tilde{I}_{i}$ are evaluated.

All of these steps are combined in the procedure MPLCubicalintegrate $(f, \operatorname{var}, n)$. The first argument is an integrand as in eq. 3.3 in a set of cubical coordinates $x_{1}, \ldots, x_{m}$ and the second argument is the last of these variables, $x_{m}$. The positive integer $n$ is the number of integrations to be computed. The procedure integrates from 0 to 1 over the variables $x_{m}, x_{m-1}, \ldots, x_{m-n+1}$ in this order.

\section{Example:}

We consider two families of integrals

$$
I_{1}(N)=\int_{0}^{1} \int_{0}^{1} d x_{1} d x_{2} f_{1}(N) \text { and } I_{2}(N)=\int_{0}^{1} \int_{0}^{1} \int_{0}^{1} d x_{1} d x_{2} d x_{3} f_{2}(N)
$$

with integrands

$$
\begin{aligned}
& f_{1}(N)=(-1)^{N} \frac{x_{1}^{N}\left(1-x_{1}\right)^{N} x_{2}^{N}\left(1-x_{2}\right)^{N}}{\left(1-x_{1} x_{2}\right)^{N+1}} \\
& f_{2}(N)=\frac{x_{1}^{N}\left(1-x_{1}\right)^{N} x_{2}^{2 N+1}\left(1-x_{2}\right)^{N} x_{3}^{N}\left(1-x_{3}\right)^{N}}{\left(1-x_{1} x_{2}\right)^{N+1}\left(1-x_{2} x_{3}\right)^{N+1}}
\end{aligned}
$$

for $N \in \mathbb{N} \cup\{0\}$.

These integrals arise, after a change of variables, from Beukers' proofs [6] of Apéry's theorems [4] on the irrationality of $\zeta(2)$ and $\zeta(3)$. The general role of period integrals on $\mathcal{M}_{0, n}$ in irrationality proofs for zeta values is worked out in [22]. MPL can be used to confirm the results

$$
\begin{aligned}
& I_{1}(N)=a_{1}(N) \zeta(2)-b_{1}(N) \\
& I_{2}(N)=2 a_{2}(N) \zeta(3)-2 b_{2}(N)
\end{aligned}
$$

where the sequences of numbers $a_{1}(N), b_{1}(N)$ satisfy the recurrence relation

$$
u_{1}(N)=N^{-2}\left(\left(11 N^{2}-11 N+3\right) u_{1}(N-1)+(N-1)^{2} u_{1}(N-2)\right)
$$

with initial conditions $a_{1}(0)=1, a_{1}(1)=3, b_{1}(0)=0, b_{1}(1)=5$ and the numbers $a_{2}(N), b_{2}(N)$ satisfy

$$
u_{2}(N)=N^{-3}\left(\left(34 N^{3}-51 N^{2}+27 N-5\right) u_{2}(N-1)-(N-1)^{3} u_{2}(N-2)\right)
$$

with $a_{2}(0)=1, a_{2}(1)=5, b_{2}(0)=0, b_{2}(1)=6$.

For example, setting the first argument $f$ of the procedure MPLCubicalintegrate equal to 
$f_{2}(4)$, we compute 3

$$
\begin{aligned}
& \text { MPLCubical Integrate (f, x [3] , 3) ; } \\
& -11424695 / 144+66002 \zeta(3)
\end{aligned}
$$

in agreement with eq. 13. Here the program integrates over $x_{3}, x_{2}, x_{1}$. Intermediate results can be obtained by setting the third argument equal to 1 or 2 .

\section{Integration over Feynman parameters}

In this section, we introduce procedures to express a very general class of integrals in terms of integrals of the form of eq. 11, such that the procedures of section 2 can be used for their computation. For the sake of concreteness, we assume an application to Feynman integrals of perturbative quantum field theory, but the procedures may apply to integrals of a different context as well.

We consider integrals of the form

$$
I_{F}=\int_{0}^{\infty} d \alpha_{j} \frac{\prod_{Q_{i} \in Q} Q_{i}^{\delta_{i}} L_{w}\left(\alpha_{j}\right)}{\prod_{P_{i} \in \mathcal{P}} P_{i}^{\beta_{i}}}
$$

where $Q, \mathcal{P} \subset \mathbb{Q}\left[\alpha_{1}, \ldots, \alpha_{j}, \ldots, \alpha_{N}\right]$ are sets of irreducible polynomials, all $\delta_{i}, \beta_{i} \in \mathbb{N} \cup\{0\}$ and $L_{w}\left(\alpha_{N}\right)$ is a hyperlogarithm, given by a word $w$ in differential 1-forms in

$$
\Omega_{F}=\left\{\frac{d \alpha_{j}}{\alpha_{j}}, \frac{d \alpha_{j}}{\alpha_{j}-\rho_{i}} \text { where } \rho_{i}=-\frac{\left.P_{i}\right|_{\alpha_{j}=0}}{\frac{\partial P_{i}}{\partial \alpha_{j}}}, P_{i} \in \mathcal{P}\right\} .
$$

Let us compare the integrals of eq. 14 to the ones of eq. 11, The essential difference is between the sets $\mathcal{P}_{C}$ and $\mathcal{P}$, i.e. the polynomials in the denominator of the integrand and defining the denominators of the differential 1-forms of the iterated integrals in the numerator of the integrand. While $\mathcal{P}_{C}$ is a very specific set of polynomials (eq. 12), we will allow the set $\mathcal{P}$ to contain polynomials of a much more general type.

In the following, we consider successive integrations of the type of eq. 14 over several variables $\alpha_{\sigma(1)}, \alpha_{\sigma(2)}, \ldots, \alpha_{\sigma(N)}$ with respect to some permutation $\sigma$ of $\{1, \ldots, N\}$. Let $\mathcal{P}^{(1, \ldots, i)}$ denote the set of polynomials which plays the role of $\mathcal{P}$ in eq. 14 and 15 for integrands after the first $i$ integrations. In the following, let $\mathcal{P}$ denote the first such set, before any integrations. Let us call a polynomial $a t$ most linear in some variable, if its degree in this variable is either 0 or 1.

Integrals of this type can be computed with MPL, if the following conditions are satisfied:

\footnotetext{
${ }^{3}$ Cubical coordinates are declared internally before each integration, therefore it is not necessary to use MPLCoordinates here.
} 
1. The integral has to be finite. Of course, this condition is failed by many Feynman integrals. However, there are powerful methods to express divergent Feynman integrals in terms of finite integrals, e.g. [52, 50, 24]. In the following we assume that the integral under consideration is either a finite Feynman integral or one of the finite integrals arising from such a procedure.

2. The integral has to be linearly reducible [18], i.e. there is an order of integrations $\sigma$ such that every polynomial in $\mathcal{P}^{(\sigma(1), \ldots, \sigma(i))}$ is at most linear in $\alpha_{\sigma(i+1)}$ for all $i=0, \ldots, N-1$. Linear reducibility can be checked before the integration procedure by the algorithms of [18, 19] which are implemented in MPL as discussed in section 4.1 .

3. Our implementation of the computation of limits (section 3.2) implies, that the integral has to be unramified (as defined in [18]) and $\mathcal{P}$ has to satisfy a condition, which we will call properly ordered. In section 4.2, we recall the aspects of our discussion of [13], which make these conditions precise.

Procedures to check these conditions and to compute an integral which satisfies the conditions are introduced below.

\subsection{Polynomial reduction}

For each permutation $\sigma$ on $\{1, \ldots, N\}$, Brown's polynomial reduction algorithms [18, 19] construct sequences

$$
S^{\{\sigma(1)\}}, S^{\{\sigma(1), \sigma(2)\}}, \ldots, S^{\{\sigma(1), \sigma(2), \ldots, \sigma(i)\}},
$$

with $i \leq N$, of sets of irreducible polynomials, such that

$$
\mathcal{P}^{(\sigma(1), \ldots, \sigma(i))} \subseteq S^{\{\sigma(1), \ldots, \sigma(i)\}}, i \in\{1, \ldots, N\} .
$$

If $S^{\{\sigma(1), \ldots, \sigma(i)\}}$ contains a polynomial of degree greater than 1 in $\alpha_{\sigma(i+1)}$ then the set $S^{\{\sigma(1), \ldots, \sigma(i), \sigma(i+1)\}}$ with respect to the order given by $\sigma$ is not constructed. The initial set $\mathcal{P}$ is called linearly reducible, if there is a $\sigma$ such that the algorithm succeeds to construct a full sequence $S^{\{\sigma(1)\}}, \ldots, S^{\{\sigma(1), \ldots, \sigma(N)\}}$.

We briefly recall the construction of the sets $S^{\{\sigma(1), \ldots, \sigma(i)\}}$. Let $S \subset \mathbb{Q}\left[\alpha_{1}, \ldots, \alpha_{N}\right]$ and let $S_{\text {irred. }}$. denote the set of all irreducible factors of all polynomials in $S$, disregarding constants. Adapting the notation of [53] we define

$$
[P, 0]_{i}=\left.P\right|_{\alpha_{i}=0},[P, \infty]_{i}=\left\{\begin{array}{ll}
\frac{\partial P}{\partial \alpha_{i}} & \text { if } \frac{\partial P}{\partial \alpha_{i}} \neq 0, \\
\left.P\right|_{\alpha_{i}=0} & \text { otherwise, }
\end{array} \text { and }\left[P_{j}, P_{k}\right]_{i}=\left.\frac{\partial P_{j}}{\partial \alpha_{i}} P_{k}\right|_{\alpha_{i}=0}-\left.\frac{\partial P_{k}}{\partial \alpha_{i}} P_{j}\right|_{\alpha_{i}=0}\right.
$$


For sets $S$ all of whose polynomials are at most linear in $\alpha_{i}$, we define the simple reduction [18] of $S$ with respect to $\alpha_{i}$ by

$$
S_{i}=\left\{[P, 0]_{i},[P, \infty]_{i}: P \in S\right\}_{\text {irred. }} \cup\left\{\left[P_{j}, P_{k}\right]_{i}: P_{j}, P_{k} \in S\right\}_{\text {irred. }} .
$$

One defines the Fubini reduction [18] of $\mathcal{P}$ with respect to $\sigma$ by

$$
\begin{aligned}
S^{\emptyset} & =\mathcal{P}, \\
S^{\{\sigma(1), \ldots, \sigma(i)\}} & =\cap_{k \in\{\sigma(1), \ldots, \sigma(i)\}} S_{k}^{\{\sigma(1), \ldots, \sigma(i)\} \backslash\{k\}},
\end{aligned}
$$

where on the right-hand side only the sets $S^{\{\sigma(1), \ldots, \sigma(i)\} \backslash\{k\}}$ are considered, which are defined by simple reduction and whose polynomials are at most linear $\alpha_{k}$. Disregarding monomials and constants, these sets satisfy eq. 16. Moreover, for every permutation $\lambda$ on $\{\sigma(1), \ldots, \sigma(i)\}$ we have

$$
\mathcal{P}^{(\lambda(\sigma(1)), \ldots, \lambda(\sigma(i)))} \subseteq S^{\{\sigma(1), \ldots, \sigma(i)\}}, i \in\{1, \ldots, N\}
$$

assuming that both sides of this relation exist.

A more refined upper bound was defined in [19] by introducing compatibility graphs. Here we adapt the version defined in [53]. The above simple reduction is extended to a pair $(S, C)$ where $S$ is a set of irreducible polynomials and $C$ is the set of edges of a graph, whose vertices are the polynomials in $S$. A pair of two distinct polynomials in $S$ is called compatible, if there is an edge between the corresponding two vertices in $C$. For a set $S$ with all polynomials at most linear in $\alpha_{i}$ one defines

$$
\bar{S}_{i}=\left\{[P, 0]_{i},[P, \infty]_{i}: P \in S\right\}_{\text {irred. }} \cup\left\{\left[P_{j}, P_{k}\right]_{i}: \text { compatible pairs } P_{j}, P_{k} \in S\right\}_{\text {irred. }} .
$$

In the corresponding compatibility graph $C_{i}$ the vertices are given by $\bar{S}_{i}$ and the edges are between all pairs of distinct irreducible factors of $\left[P_{j}, P_{k}\right]_{i} \cdot\left[P_{k}, P_{l}\right]_{i} \cdot\left[P_{l}, P_{j}\right]_{i}$ for every mutually compatible $P_{j}, P_{k}, P_{l} \in S \cup\{0, \infty\}$. The auxiliary terms 0 and $\infty$ are each considered compatible with every element of $S \cup\{0, \infty\}$. The initial pair of this reduction is $\left(\mathcal{P}, K_{\mathcal{P}}\right)$, where $K_{\mathcal{P}}$ is the complete graph whose vertices are the polynomials in $\mathcal{P}$. We have $\bar{S}_{i} \subseteq S_{i}$ by construction. It was proven in [53], that the sequence obtained by this extended simple reduction is an upper bound for the sets $\mathcal{P}^{(\sigma(1), \ldots, \sigma(i))}$ as in eq. 16 . 
In analogy to the Fubini reduction, we furthermore define

$$
\begin{aligned}
\left(S^{\emptyset}, C^{\emptyset}\right)= & \left(\mathcal{P}, K_{\mathcal{P}}\right), \\
\left(\bar{S}^{\{\sigma(1), \ldots, \sigma(i)\}}, C^{\{\sigma(1), \ldots, \sigma(i)\}}\right)= & \left(\cap_{k \in\{\sigma(1), \ldots, \sigma(i)\}} \bar{S}_{k}^{\{\sigma(1), \ldots, \sigma(i)\} \backslash\{k\}},\right. \\
& \left.\cap_{k \in\{\sigma(1), \ldots, \sigma(i)\}} C_{k}^{\{\sigma(1), \ldots, \sigma(i)\} \backslash\{k\}}\right)
\end{aligned}
$$

where the intersection $G_{1} \cap G_{2}$ of graphs $G_{1}, G_{2}$ is a graph consisting only of the vertices and the edges which belong to both of the graphs. Note that this construction includes the above Fubini reduction, if we replace every graph in eq. 18 with the corresponding complete graph. Therefore we have

$$
\bar{S}^{\{\sigma(1), \ldots, \sigma(i)\}} \subseteq S^{\{\sigma(1), \ldots, \sigma(i)\}}
$$

by construction. An explicit proof that the sets $\bar{S}^{\{\sigma(1), \ldots, \sigma(i)\}}$ satisfy eq. 16 is missing at this point. However, these sets have served as correct upper bounds in all known cases so far (see the remark in sec. 3.6.6 of [53]).

The program MPL provides the procedure

$>$ MPLPolynomialReduction (S, L) ;

where the first argument is the list of polynomials to be reduced and the second argument is the list of all variables with respect to which the reduction shall be computed. In a typical Feynman integral computation, $S$ would contain the Symanzik polynomials and $L$ would be the list of Feynman parameters (cf. the example in appendix A). The procedure returns a list where each entry corresponds to a pair $\left(\bar{S}^{\{\sigma(1), \ldots, \sigma(i)\}}, C^{\{\sigma(1), \ldots, \sigma(i)\}}\right)$ of the above reduction. For example, a typical entry of this list would be

$$
\left[\left[\alpha_{1}, \alpha_{4}\right],\left[\alpha_{2} \alpha_{3}+1, \alpha_{2}-\alpha_{3}, \alpha_{2} \alpha_{5}+\alpha_{3}\right],[\{1,2\},\{1,3\}]\right]
$$

Every entry contains three lists. The first list contains the reduced variables, the second list contains the polynomials in the remaining variables (disregarding monomials and constants) and the third list contains the edges of the compatibility graph, in terms of pairs of the numbers of compatible polynomials with respect to the given list. In this sense, the above example stands for the pair $\left(\bar{S}^{\{1,4\}}, C^{\{1,4\}}\right)$ with $\bar{S}^{\{1,4\}}=\left\{\alpha_{2} \alpha_{3}+1, \alpha_{2}-\alpha_{3}, \alpha_{2} \alpha_{5}+\alpha_{3}\right\}$ and where the first polynomial of this list is compatible with the second one and the third one.

A global, boolean variable COMPATIBILITY_GRAPH is set true by default. If the user sets this variable to false, the procedure MPLPolynomialReduction returns a list corresponding to the sets $S^{\{\sigma(1), \ldots, \sigma(i)\}}$ of the Fubini reduction (eq. 17).

In both cases, the polynomial reduction algorithm considers all permutations on $\{1, \ldots, N\}$. If 
the reduction (by at least one of both algorithms) contains a complete sequence

$$
S^{\{\sigma(1)\}}, \ldots, S^{\{\sigma(1), \ldots, \sigma(N)\}}
$$

(or with $\bar{S}$ instead of $S$ ) for one such permutation $\sigma$, linear reducibility is satisfied.

If this is not the case, the algorithm has stopped the construction of sets at certain stages, because of polynomials which are non-linear in some $\alpha_{i}$. There are several possible scenarios. Firstly, these polynomials might be spurious, i.e. they belong to the upper bounds generated by the algorithm, but might not appear in the actual integrands. In this (rather unlikely) case, the integral can still be computed. Secondly, the problematic polynomials may belong to $\mathcal{P}^{(\sigma(1), \ldots, \sigma(i))}$, but it may be possible to restore linearity in some variable by an appropriate change of variables. Such examples 4 are discussed in [52, 53]. These changes of variables are not implemented in MPL so far. Thirdly, it may be the case that such changes of variables do not exist and that neither iterated integrals on $\mathcal{M}_{0, n}$ nor hyperlogarithms are sufficient to express the given integral $[$.

\subsection{Changes of variables}

Let us assume an integrand of the type of eq. 14 whose polynomials and hyperlogarithms depend on $N$ Feynman parameters $\alpha_{1}, \ldots, \alpha_{N}$ and on $M$ kinematical invariants $s_{1}, \ldots, s_{M}$ (possibly including squared particle masses). Furthermore, let these kinematical invariants be defined such that in the momentum region where we want to compute the integral, all $s_{i}$ take only non-negative, real values. The most convenient way to compute with such variables in MPL is to treat them like additional Feynman parameters, which are not integrated out. In this sense, let us define the $N+M$ generalized Feynman parameters $a_{i}$ by

$$
a_{i}=s_{i} \text { and } a_{M+j}=\alpha_{j} \text { for } i=1, \ldots, M \text { and } j=1, \ldots, N
$$

The transformations of integrands from Feynman parameters to cubical coordinates and back are discussed in detail in section 4 of [13], without explicitly mentioning kinematical invariants. However, the discussion in [13] is easily extended to include these dependences by simply replacing the Feynman parameters by the generalized Feynman parameters. Making this point explicit here, we recall the remaining conditions on the integrand.

Let us assume that the set of polynomials $\mathcal{P}=\left\{P_{1}, \ldots, P_{m}\right\}$ of the integrand is linearly reducible. Let $T=N+M$ and let us number the (generalized) Feynman parameters such that from left to right

\footnotetext{
${ }^{4}$ An example for this case is the massless graph $K_{4}$ with four on-shell legs, which was found to be irreducible with respect to Feynman parameters in [14] but computable in terms of harmonic polylogarithms in [43]. A change of variables restoring linear reducibility was found in [52].

${ }^{5}$ In such cases, the use of elliptic polylogarithms as in the computations [11, 10, 2, 3] may be appropriate.
} 
$a_{T}, a_{T-1}, \ldots, a_{M+1}$ is the desired order of integrations. Following the algorithm of [13], MPL introduces $m$ cubical coordinates $x_{1}, \ldots, x_{m}$ by a homomorphism $\phi^{\star}: \mathbb{Q}\left(x_{1}, \ldots, x_{m}\right) \rightarrow \mathbb{Q}\left(a_{1}, . ., a_{T}\right)$ and expresses the integrand in terms of integrals of the type 11, such that all polynomials and hyperlogarithms depending on the next integration variable $a_{T}=\alpha_{N}$ are expressed in terms of cubical coordinates. After integrating over $x_{m}$, the result is expressed in terms of hyperlogarithms in Feynman parameters again. As explained in detail in [13], these functions $\eta$ have to satisfy a certain condition at the tangential basepoint corresponding to consecutive limits, for which we define the short-hand notation $\lim _{a \rightarrow 0_{T-1}}$ by

$$
\lim _{a \rightarrow 0_{T-1}} \eta=\lim _{a_{1} \rightarrow 0} \ldots \lim _{a_{T-1} \rightarrow 0} \eta
$$

These limits in the space of Feynman parameters correspond to limits in cubical coordinates, in the sense that

$$
\lim _{x_{p} \rightarrow c} g=\lim _{a \rightarrow 0_{T-1}} \phi^{\star} g
$$

for any rational function $g$ in the $x$-coordinates. Here $\lim _{x_{p} \rightarrow c} g$ is the short-hand notation defined by

$$
\lim _{x_{p} \rightarrow c} g=\lim _{x_{p(1)} \rightarrow c_{p(1)}} \cdots \lim _{x_{p(m-1)} \rightarrow c_{p(m-1)}} g
$$

where $p$ is a particular permutation on $\{1, \ldots, m-1\}$ and $c_{i}=\lim _{a \rightarrow 0_{T-1}} x_{i}$ for $i=1, \ldots, m-1$. As we take limits with respect to all remaining variables here, the $c_{i}$ are real numbers.

In MPL, limits as in eq. 20 can be computed by use of the procedures discussed in section 3.2, if two conditions are satisfied. Firstly, the limit has to be computed at a corner-point of the unit-cube

$$
\mathbb{R}_{\text {cube }}^{m-1}=\left\{\left(x_{1}, \ldots, x_{m-1}\right) \in \mathbb{R}^{m-1} \mid 0 \leq x_{i} \leq 1, i=1, \ldots, m-1\right\},
$$

i.e. $c_{i} \in\{0,1\}$ for $i=1, \ldots, m-1$. Secondly, the limit has to be computed by approaching this point from inside this cube.

We introduce two conditions on the set $\mathcal{P}$, such that these conditions on the limits are satisfied. For each $P_{i} \in \mathcal{P}$ let us define $\rho_{i}=-\frac{P_{i} \mid \alpha_{N}=0}{\frac{\partial P_{i}}{\partial \alpha_{N}}}$ and consider the space of generalized Feynman parameters

$$
\mathbb{R}^{T-1}=\left\{\left(a_{1}, \ldots, a_{T-1}\right) \in \mathbb{R}^{T-1} \mid 0 \leq a_{i}, i=1, \ldots, T-1\right\} .
$$

As a first condition, let there be an open region $\Lambda \subset \mathbb{R}^{T-1}$ with all points satisfying $0 \leq a_{T-1} \ll$ $a_{N-2} \ll \ldots \ll a_{1} \ll \varepsilon$, where $x \ll y$ denotes $x<y^{K}$, for a sufficiently small number $\varepsilon$ and a sufficiently large number $K$, such that everywhere in $\Lambda$ we have 


$$
0>\rho_{m}>\rho_{1}>\rho_{2}>\ldots>\rho_{m-2}>\rho_{m-1} .
$$

Here we have already numbered the $P_{i}$ according to this unique order. If such a region $\Lambda$ exists, let us call the integral properly ordered.

The change of coordinates introduced in [13] is defined as

$$
\phi^{\star}\left(x_{m}\right)=\frac{a_{T}}{a_{T}-\rho_{m}}, \phi^{\star}\left(x_{m-1}\right)=\xi_{m-1} \text { and } \phi^{\star}\left(x_{k}\right)=\frac{\xi_{k}}{\xi_{k+1}} \text { for } 1 \leq k \leq m-2,
$$

where $\xi_{i}=1-\frac{\rho_{m}}{\rho_{i}}$ for $i=1, \ldots, m-1$. As a consequence of eq. 21, we have $0<\xi_{i}<1$ and $\xi_{i}<\xi_{i+1}$ and therefore $0<x_{i}<1, i=1, \ldots, m-1$, everywhere in $\Lambda$. Therefore, if the integral is properly ordered, the limit eq. 20 is approached from inside $\mathbb{R}_{\text {cube }}^{m-1}$.

As a second condition, we assume that the set $\left\{\rho_{1}, \ldots, \rho_{m}\right\}$ is unramified [18], i.e.

$$
\lim _{a \rightarrow 0_{T-1}} \rho_{i} \in\{0,-1, \infty\} \text { for } i=1, \ldots, m .
$$

In this case, we also call the integral unramified. Together with the condition to be properly ordered, this implies the desired condition $c_{i} \in\{0,1\}$ for $i=1, \ldots, m-1$.

Note that it may strongly depend on the choice of kinematical invariants and the region of the momentum space, whether these conditions are satisfied. We also note, that while the choice of the order of $a_{T}, \ldots, a_{M+1}$ is at least partially restricted by the condition of linear reducibility, the order of the kinematical invariants $a_{M}, \ldots, a_{1}$ is arbitrary. If this freedom in the choice of the orders and in the choice of kinematical invariants can not be exploited to write the integrand in a form such that proper ordering and unramifiedness are satisfied, the use of iterated integrals on $\mathcal{M}_{0, n}$ or hyperlogarithms may still be possible.

\subsection{The computation of Feynman integrals with MPL}

Let us now turn to the computation of a finite integral in Feynman parameters with MPL. We choose a letter to denote the generalized Feynman parameters, say $a_{i}$. In the Maple worksheet, this choice is declared by the Maple command

$$
>\operatorname{defform}(a=0) \text { : }
$$

From here on, all a $[i]$ are treated as variables and all $d(a[i])$ are treated as differential 1-forms by Maple.

For a given set $\mathcal{P}$ of polynomials in the integrand of the type of eq. 14 we check with MPLPolynomialReduction whether the integrand is linearly reducible (see section 4.1). Here let 
us assume that linear reducibility is satisfied. For convenience, we number the integration variables such that an allowed order of integrations is $a_{T}, a_{T-1}, \ldots, a_{M+2}, a_{M+1}$. Furthermore we number the $M$ kinematical invariants according to some arbitrary order. Based on these orders, we define the vector of all generalized Feynman parameters $a=\left(a_{T}, \ldots, a_{M+1}, a_{M}, \ldots, a_{1}\right)$.

The question remains, whether the integral is furthermore properly ordered and unramified with respect to the order in $a$. This can be checked with the MPL command

$$
\text { >MPLCheckOrder(reduction, a [1..k], a); }
$$

Here the first argument is the list generated by MPLPolynomialReduction, the second argument is a list of the variables in $a$ to be integrated out and the last argument is the list $a=[a[\mathrm{~T}], \ldots, \mathrm{a}[1]]$ of all components of $a$. In both lists, the entries are ordered according to the chosen vector $a$.

If the latter procedure confirms the conditions to be satisfied, we can use the order of $a$ to compute the integral6. This is done with the command

$>$ MPLFeynmanIntegrate (integrand, a [1..k], a);

Here the first argument is the integrand of the type of eq. 14. Note that the numerator of the integrand has to be a linear combination of logarithms and hyperlogarithms in bar-notation (using bar (...)), if such functions occur. The second and third argument are the same as in the previous procedure. We obtain the final result by integrating over $k=T-M$ variables and intermediate results by chosing $k<T-M$. The results are given in terms of hyperlogarithms $L$ in the remaining generalized Feynman parameters, vanishing in the $\operatorname{limit} \lim _{a \rightarrow 0_{T}} L$ by definition.

The Feynman parametric version of the integral is sometimes expressed with a $\delta(H)$ in the integrand, where $H$ is some hypersurface in the space of the integration variables, to be chosen freely, according to the famous Cheng-Wu theorem [28]. We always choose $H=1-a_{M+1}$, which implies that we integrate over the first $k=T-M-1$ variables with the above command and take

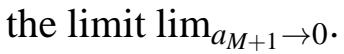

Detailed examples of Feynman integral computations with MPL are presented in the manual and in appendix A.

\section{Conclusions}

We have introduced the computer program MPL for computations with iterated integrals on moduli spaces of curves of genus zero with $n$ ordered marked points with Maple. The program includes procedures for the computation of the symbol map, the unshuffle map, the total differentiation, the

\footnotetext{
${ }^{6}$ If one of the conditions fails, the freedom of choices with respect to kinematical invariants as mentioned in section 4.2 may be used to try a different $a$.
} 


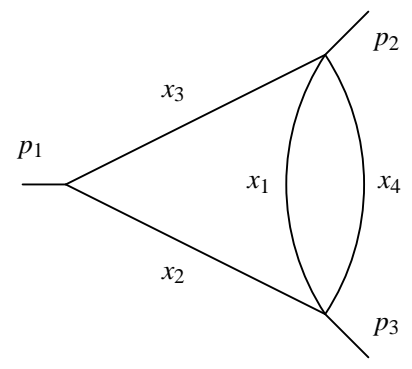

Figure 1: A two-loop triangle graph

derivation of primitives and certain limits. It provides the analytical computation of a very general class of integrals which can be understood as period integrals on the mentioned moduli spaces.

By additional procedures, deriving appropriate changes of variables, the program furthermore supports the computation of Feynman integrals. It provides the automated computation of finite, linearly reducible integrals over Feynman parameters if the order of integrations satisfies unramifiedness and properly ordered polynomials at a tangential basepoint.

\section{Acknowledgements}

I thank Francis Brown for introducing me to the subject of this article, for crucial advice at various stages of writing the program and very useful remarks on the manuscript. I am grateful to Erik Panzer for his help, particularly on the application to Feynman integrals, and also for very useful remarks on the manuscript. I am also indebted to Dirk Kreimer for his strong support and encouragement for the project. This work was partly supported by Deutsche Forschungsgemeinschaft. The graphs were drawn using [41].

\section{Appendix A: An example of a Feynman integral computation}

As an example application for the procedures introduced in section 4, let us consider the massless, off-shell Feynman graph of figure 1 whose integral was already computed in [26]. We denote the Feynman parameters by $x_{1}, x_{2}, x_{3}, x_{4}$ and the incoming external momenta by $p_{1}, p_{2}, p_{3}$. The Symanzik polynomials (see e.g. [16]) of the graph are

$$
\begin{aligned}
\mathcal{U} & =x_{1} x_{4}+\left(x_{1}+x_{4}\right)\left(x_{2}+x_{3}\right) \\
\mathcal{F} & =-p_{1}^{2} x_{2} x_{3}\left(x_{1}+x_{4}\right)-p_{2}^{2} x_{1} x_{3} x_{4}-p_{3}^{2} x_{1} x_{2} x_{4}
\end{aligned}
$$


Expressing the kinematical dependences by two variables $x_{5}$ and $x_{6}$, defined by (cf. [26])

$$
\frac{p_{2}^{2}}{p_{1}^{2}}=\left(1+x_{5}\right)\left(1+x_{6}\right) \text { and } \frac{p_{3}^{2}}{p_{1}^{2}}=x_{5} x_{6},
$$

we furthermore define a slightly modified second Symanzik polynomial

$$
\tilde{\mathcal{F}}=-\frac{\mathcal{F}}{p_{1}^{2}}=x_{2} x_{3}\left(x_{1}+x_{4}\right)+x_{1} x_{3} x_{4}\left(1+x_{5}\right)\left(1+x_{6}\right)+x_{1} x_{2} x_{4} x_{5} x_{6}
$$

and consider the Feynman integral around $D=4-2 \varepsilon$ dimensions

$$
I=\int_{x} \delta(H) \mathcal{U}^{3 \varepsilon-2} \tilde{\mathcal{F}}^{-2 \varepsilon}
$$

Here we omitted a trivial prefactor $\left(-p_{1}^{2}\right)^{2 \varepsilon} \Gamma(2 \varepsilon)$ and we introduced $\int_{x}$ as a short-hand notation for $\prod_{i=1}^{4}\left(\int_{0}^{\infty} d x_{i}\right)$. In the following, we consider the region in momentum space, where $x_{5}>0, x_{6}>0$, i.e. the set $\left\{x_{1}, \ldots, x_{6}\right\}$ are generalized Feynman parameters in the sense of section 4.2. For the hyperplane $H$ we still have a freedom of a choice to be made below.

Let us at first express this divergent integral in terms of finite ones. Following Panzer's strategy of analytic regularization [52], we define a differential operator

$$
D_{\left\{x_{1}, x_{4}\right\}}=\frac{1}{\varepsilon}\left((\varepsilon-2)-x_{1} \frac{\partial}{\partial x_{1}}-x_{4} \frac{\partial}{\partial x_{4}}\right)
$$

and obtain $I=\frac{1}{\varepsilon} \tilde{I}$ with

$$
\begin{aligned}
\tilde{I} & =\varepsilon \int_{x} \delta(H) D_{\left\{x_{1}, x_{4}\right\}}\left(\mathcal{U}^{3 \varepsilon-2} \tilde{\mathcal{F}}^{-2 \varepsilon}\right) \\
& =\int_{x} \delta(H) \frac{\mathcal{U}^{3 \varepsilon-2} \tilde{\mathcal{F}}^{-2 \varepsilon}(P+\varepsilon Q)}{\mathcal{U} \mathcal{F}}
\end{aligned}
$$

Here $P$ and $Q$ are polynomials in $x_{1}, \ldots, x_{6}$, implicitly defined by this relation. The integral $\tilde{I}$ is finite at $\varepsilon=0$. By expanding its integrand at this point we obtain

$$
\begin{aligned}
\tilde{I} & =\tilde{I}^{(0)}+\varepsilon \tilde{I}^{(1)}+\varepsilon^{2} \tilde{I}^{(2)}+O\left(\varepsilon^{3}\right) \\
\tilde{I}^{(0)} & =\int_{x} \delta(H) \frac{P}{\mathcal{U}^{3} \tilde{\mathcal{F}}}, \\
\tilde{I}^{(1)} & =\int_{x} \delta(H) \frac{Q+P(3 \ln (\mathcal{U})-2 \ln (\tilde{\mathcal{F}}))}{\mathcal{U}^{3} \tilde{\mathcal{F}}}, \\
\tilde{I}^{(2)} & =\int_{x} \delta(H) \frac{Q(6 \ln (\mathcal{U})-4 \ln (\tilde{\mathcal{F}}))+P\left(9 \ln (\mathcal{U})^{2}+4 \ln (\tilde{\mathcal{F}})^{2}-12 \ln (\mathcal{U}) \ln (\tilde{\mathcal{F}})\right)}{2 \mathcal{U}^{3} \tilde{\mathcal{F}}} .
\end{aligned}
$$


In the Maple worksheet, we begin the computation with $>\operatorname{defform}(x=0)$ :

From here on, Maple considers every x [i ] as a variable and every d (x [i]) as a differential 1-form. In order to check, whether the integrals $\tilde{I}^{(0)}, \tilde{I}^{(1)}, \tilde{I}^{(2)}$ can be computed with MPL, we begin with a polynomial reduction of the set $S=\{\mathcal{U}, \tilde{\mathcal{F}}\}$. The reduction is obtained by

$$
>\mathrm{R}:=\text { MPLPolynomialReduction }([\mathrm{U}, \mathrm{F}],[\mathrm{x}[1], \mathrm{x}[2], \mathrm{x}[3], \mathrm{x}[4]]) \text {; }
$$

The list in the first argument contains the polynomials to be reduced and the second argument is the list of integration variables in arbitrary order. The output $\mathrm{R}$ of this command is a list where each entry corresponds to a $\bar{S}^{\{\sigma(1), \ldots, \sigma(k)\}}$ as explained in section 4.1, It is easy to check, that there are permutations $\sigma$ such that a complete reduction

$$
\left\{\bar{S}^{\{\sigma(1)\}}, \bar{S}^{\{\sigma(1), \sigma(2)\}}, \bar{S}^{\{\sigma(1), \sigma(2), \sigma(3)\}}, \bar{S}^{\{\sigma(1), \sigma(2), \sigma(3), \sigma(4)\}}\right\}
$$

is contained in this list. One of these permutations defines the order $x_{1}, x_{4}, x_{3}, x_{2}$. Defining an order on all generalized Feynman parameters accordingly, we choose $a=\left(x_{1}, x_{4}, x_{3}, x_{2}, x_{5}, x_{6}\right)$ and check, whether the integrand is unramified and properly ordered with respect to this order by

$$
\begin{aligned}
& >a:=[x[1], x[4], x[3], x[2], x[5], x[6]]: \\
& >\operatorname{MPLCheckOrder}(R, a[1 \ldots 4], a) ;
\end{aligned}
$$

As all conditions are satisfied, the integral can be computed with respect to the chosen order. Now we choose the hyperplane $H$ to be $1-x_{2}=0$.

We have to express all (products of) hyperlogarithms in the integrand as linear combinations of iterated integrals in bar-notation. For the given example, it is sufficient to re-write the integrands of eq. 24 by use of

$$
\ln (X)=\left[\frac{d(X)}{X}\right] \text { and } \ln (X) \ln (Y)=\left[\frac{d(X)}{X} \mid \frac{d(Y)}{Y}\right]+\left[\frac{d(Y)}{Y} \mid \frac{d(X)}{X}\right]
$$

where the iterated integrals are expressed by use of bar (..) in the Maple worksheet (see section 2.1). With these integrands, we can now compute the first three integrations with $>$ MPLFeynmanIntegrate (integrand, a [1..3], a) ;

The output is the result after integration over $x_{1}, x_{4}, x_{3}$ and according to our choice of $H$, we finally set $x_{2}=1$ in this result. Here, all of these results are rational functions of $x_{2}$, so we can simply use 7

\footnotetext{
${ }^{7}$ If one of the results would be an iterated integral depending on $x_{2}$, we would apply the command MPLLimit instead.
} 
$>\operatorname{eval}\left(\frac{\circ}{0} \mathrm{x}[2]=1\right)$;

We obtain the results

$$
\begin{aligned}
\tilde{I}^{(0)=} & 1, \\
\tilde{I}^{(1)}= & 5, \\
\tilde{I}^{(2)=} & \frac{1}{x_{6}-x_{5}}\left(2 x_{5}\left(1+x_{6}\right)\left(\left[\frac{d\left(x_{6}\right)}{1+x_{6}}\right]\left[\frac{d\left(x_{5}\right)}{x_{5}}\right]+\left[\frac{d\left(x_{5}\right)}{x_{5}} \mid \frac{d\left(x_{5}\right)}{1+x_{5}}\right]\left[\frac{d\left(x_{5}\right)}{1+x_{5}} \mid \frac{d\left(x_{5}\right)}{x_{5}}\right]\right)\right. \\
& \left.-2 x_{6}\left(1+x_{5}\right)\left(\left[\frac{d\left(x_{5}\right)}{1+x_{5}}\right]\left[\frac{d\left(x_{6}\right)}{x_{6}}\right]+\left[\frac{d\left(x_{6}\right)}{x_{6}} \mid \frac{d\left(x_{6}\right)}{1+x_{6}}\right]+\left[\frac{d\left(x_{6}\right)}{1+x_{6}} \mid \frac{d\left(x_{6}\right)}{x_{6}}\right]\right)\right) \\
& -3 \zeta(2)+19 .
\end{aligned}
$$

Here all bar-terms can be interpreted as logarithms and dilogarithms in the kinematical invariants $x_{5}, x_{6}$.

The same integrals are as well used as an example in the tutorial-worksheet of Panzer's program HyperInt [54] and the reader may find it instructive to compare the expressions. MPL provides the command MPLHlogToBar to automatically express the function Hlog of HyperInt in terms of our bar-notation.

As a final remark, we briefly recall, why MPL in general returns the result of integrations over Feynman parameters in terms of hyperlogarithms. Internally, the program introduces cubical coordinates for each integration variable and computes the integrals with the procedures introduced in section 2. However, as we are interested to see the result of the integrations expressed in terms of the kinematical invariants and possibly remaining Feynman parameters (such as $x_{2}$ here), which we usually cannot interpret as cubical coordinates of some $\mathcal{M}_{0, n}$, the program has to return the result in terms of hyperlogarithms in these parameters. If the integrand in Feynman parameters and kinematical invariants would already be of the form of eq. 11 from the beginning, we could simply declare these parameters as cubical coordinates and compute the integrations by use of MPLCubical Integrate as in section 3.3. Usually, due to the complexity of Symanzik polynomials, this is not the case and a change of variables is needed.

\section{Appendix B: A quick introduction to the spaces $\mathcal{M}_{0, n}$}

In the literature on Feynman integrals, moduli spaces of curves are not a very common topic. In this article we have frequently mentioned "moduli spaces $\mathcal{M}_{0, n}$ of curves of genus zero with $n$ ordered, marked points", without explaining what this phrase means. Without assuming familiarity with moduli spaces, we have introduced the corresponding class of iterated integrals by an ad-hoc definition in section 2. However, it may be more satisfying to see, how the differential 1-forms in 
cubical coordinates arise and what the underlying spaces look like. Here we try to give a very basic introduction.

Speaking very generally, a moduli space is a device for the classification of certain objects with respect to equivalence relations. For a given set of objects, a moduli space is constructed, such that each point of the space corresponds to an equivalence class in the set. (See [42] for a very general discussion of such moduli problems.) In the case of moduli spaces of curves, the objects are algebraic curves and each point of the moduli space corresponds to an isomorphism class of such curves.

In order to keep technicalities at a minimum here, we make use of the fact, that there is a canonical bijection between the isomorphism classes of algebraic curves and the isomorphism classes of Riemann surfaces (see e.g. [51]). As a consequence, the moduli spaces of algebraic curves and of Riemann surfaces are the same, and we restrict ourselves to the terminology of Riemann surfaces here. We recall that a Riemann surface is a connected, complex analytic manifold of (complex) dimension one and its genus is, pictorially speaking, the number of its 'handles', i.e. 0 for a sphere, 1 for a torus, 2 for a frame of a pair of glasses and so on.

It is instructive to consider the case of genus 1 at first. For every Riemann surface $S$ of genus 1 there is a complex number $j(S)$ (the $j$-invariant of elliptic curves) such that two Riemann surfaces $S_{1}, S_{2}$ are isomorphic, if and only if $j\left(S_{1}\right)=j\left(S_{2}\right)$. In other words, for each isomorphism class of Riemann surfaces of genus 1 , there is a unique complex number. Moreover it is known that for any $\lambda \in \mathbb{C}$, there is an isomorphism class $\mathcal{C}$ of such curves, such that $j(S)=\lambda$ for $S \in \mathcal{C}$. Therefore the moduli space $\mathcal{M}_{1}$ of curves (or Riemann surfaces) of genus 1 is simply the complex plane $\mathbb{C}$, parametrized by $j$. Usually this moduli space is viewed as the affine line $\mathbb{A}_{j}^{1}$.

The case of genus 0 is even more simple. Every Riemann surface of genus 0 is isomorphic to the Riemann sphere $\mathbb{C} \cup\{\infty\}$. Therefore there is only one isomorphism class and the moduli space $\mathcal{M}_{0}$ of curves (or Riemann surfaces) of genus 0 is only one point.

At low genus, more interesting moduli spaces can be constructed by adding data to the Riemann surfaces. One way to do so is by marking some of their points. In the case of genus 0 , let us consider $n$ distinct, ordered points $z_{1}, z_{2}, \ldots, z_{n}$ on a Riemann sphere $S$. By saying that these points are marked, we say $S$ with these marked points is only isomorphic to a Riemann sphere $S^{\prime}$ with ordered, marked points $z_{1}^{\prime}, z_{2}^{\prime}, \ldots, z_{n}^{\prime}$, if an isomorphism between these Riemann shperes exists, which maps $z_{i}$ to $z_{i}^{\prime}$ for $i=1, \ldots, n$. Due to this restriction, there will clearly be such spheres which are not isomorphic to each other and we obtain non-trivial moduli spaces.

We recall that an automorphism is an isomorphism of a manifold onto itself. For the construction of moduli spaces of curves of genus 0 , the isomorphisms defining the classes corresponding to the points of these spaces are the automorphisms of the Riemann sphere. These are the Möbius 
transformations

$$
z \mapsto \frac{\alpha z+\beta}{\gamma z+\delta} \text { with } \alpha, \beta, \gamma, \delta \in \mathbb{C}, \alpha \delta-\beta \gamma \neq 0
$$

which form the Möbius group denoted $\mathrm{PGL}_{2}(\mathbb{C})$.

Now, on a formal level, we have all ingredients to define the moduli space $\mathcal{M}_{0, n}$ of curves of genus 0 with $n$ ordered, marked points:

$$
\mathcal{M}_{0, n}(\mathbb{C})=\left\{\left(z_{1}, \ldots, z_{n}\right) \in \mathbb{C}^{n} \cup\{\infty\} \text { distinct }\right\} / \operatorname{PGL}_{2}(\mathbb{C})
$$

Here it is constructed as a space of $n$ complex coordinates, given by the marked points on the Riemann sphere $\mathbb{C} \cup\{\infty\}$, modulo the automorphisms. Due to the condition that all points are distinct, $z_{i} \neq z_{j}$ for $i, j=1, \ldots, n$, all diagonals are excluded from this space.

In this definition, due to the division by the automorphisms $\mathrm{PGL}_{2}(\mathbb{C})$, it may still not be obvious, what this space really looks like. In fact, there is only one property of $\mathrm{PGL}_{2}(\mathbb{C})$ which we have to understand: For any Riemann sphere with $n$ ordered, marked points, there is an isomorphism in $\mathrm{PGL}_{2}(\mathbb{C})$ which maps three of the marked points to the points 0,1 and $\infty$. As a consequence, if we choose $n \leq 3$, again all spheres are isomorphic and we obtain a trivial moduli space with only one point.

The first non-trivial space is $\mathcal{M}_{0,4}$. We can map three of the marked points to $0,1, \infty$ by an element of $\mathrm{PGL}_{2}(\mathbb{C})$. The fourth point is allowed to take every complex value except these three, and defines a new isomorphism class for each new value. In this sense, the fourth point parametrizes the moduli space, which therefore has one complex dimension. Introducing one further marked point we obtain $\mathcal{M}_{0,5}$ as a space parametrized by two complex variables, excluding the points where they are equal to $0,1, \infty$ or equal to each other.

Generalizing this concept to $n$ marked points, we use the mentioned property of $\mathrm{PGL}_{2}(\mathbb{C})$ to always set $z_{1}=0, z_{n-1}=1, z_{n}=\infty$ and we introduce the so-called simplicial coordinates

$$
t_{1}=z_{2}, t_{2}=z_{3}, \ldots, t_{n-3}=z_{n-2} .
$$

In terms of these new coordinates, we obtain

$$
\mathcal{M}_{0, n} \cong\left\{\left(t_{1}, \ldots, t_{n-3}\right) \in \mathbb{C}^{n-3} \mid t_{i} \neq t_{j} \text { and } t_{i} \neq\{0,1\} \text { for } i, j=1, \ldots, n-3\right\}
$$

This construction is already suitable for practical computations. The division by $\mathrm{PGL}_{2}(\mathbb{C})$ has been made explicit and we see, that the space has $n-3$ dimensions. By a further change of variables

$$
x_{1}=\frac{t_{1}}{t_{2}}, x_{2}=\frac{t_{2}}{t_{3}}, \ldots, x_{n-4}=\frac{t_{n-4}}{t_{n-3}}, x_{n-3}=t_{n-3}
$$


we finally arrive at

$$
\mathcal{M}_{0, n} \cong\left\{\left(x_{1}, \ldots, x_{n-3}\right) \in \mathbb{C}^{n-3} \mid x_{i} x_{i+1} \ldots x_{j} \neq\{0,1\} \text { for all } 1 \leq i \leq j \leq n-3\right\}
$$

The variables $x_{i}$ are the cubical coordinates used throughout this article. Due to the above condition to the products $x_{i} x_{i+1} \ldots x_{j}$ we have well-defined differential 1-forms

$$
\frac{d x_{j}}{x_{j}} \text { and } \frac{d\left(x_{i} x_{i+1} \ldots x_{j}\right)}{x_{i} x_{i+1} \ldots x_{j}-1} \text { for } 1 \leq i \leq j \leq n-3
$$

on this space.

As a final remark, let us mention that in section 3.2 we have discussed limits at points on the hypersurfaces, which are excluded from $\mathcal{M}_{0, n}$ by the above definition. In this context, we have to

work with a compactification $\overline{\mathcal{M}}_{0, n}$ of the space. For this and other advanced issues on $\mathcal{M}_{0, n}$, we refer to [17] and references therein.

\section{References}

[1] J. Ablinger, J. Blümlein, C.G. Raab and C. Schneider, Iterated Binomial Sums and their Associated Iterated Integrals, J.Math.Phys. 55 (2014) 112301, arXiv:1407.1822 [hep-th].

[2] L. Adams, C. Bogner and S. Weinzierl, The two-loop sunrise graph in two space-time dimensions with arbitrary masses in terms of elliptic dilogarithms, J.Math.Phys. 55 (2014) 10, 102301, arXiv:1405.5640 [hep-ph].

[3] L. Adams, C. Bogner and S. Weinzierl, The two-loop sunrise integral around four space-time dimensions and generalisations of the Clausen and Glaisher functions towards the elliptic case, J.Math.Phys. 56 (2015) 7, 072303, arXiv:1504.03255 [hep-ph].

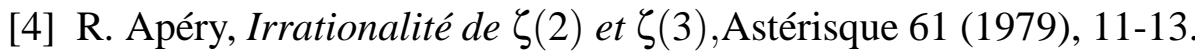

[5] V.I. Arnold, The cohomology ring of the coloured braid group, Mat. Zametki 5 (1969), 227231; Math Notes 5 (1969), 138-140.

[6] F. Beukers, A note on the irrationality of $\zeta(2)$ and $\zeta(3)$, Bull. London Math. Soc. 11 (1979), 268-272.

[7] M. Bigotte, G. Jacob, N.E. Oussous, M. Petitot and H.N. Minh, Table of the coloured zeta function, with 1 roots, up to the weight 12, URL: http://www.lifl.fr/ petitot/recherche/MzV/mzv12/.

[8] T.G. Birthwright, E.W.N. Glover and P. Marquard, Master Integrals For Massless Two-Loop Vertex Diagrams With Three Offshell Legs, JHEP0409:042, 2004, arXiv:hep-ph/0407343. 
[9] S. Bloch, E. Esnault and D. Kreimer, On motives associated to graph polynomials, Commun. Math. Phys. 267 (2006), 181-225.

[10] S. Bloch, M. Kerr and P. Vanhove, A Feynman integral via higher normal functions, arXiv:1406.2664 [hep-th].

[11] S. Bloch and P. Vanhove, The elliptic dilogarithm for the sunset graph, Journal of Number Theory 148 (2015) pp. 328-364, arXiv:1309.5865 [hep-th].

[12] C. Bogner and F. Brown, Symbolic integration and multiple polylogarithms, PoS LL2012 (2012) 053, arXiv:1209.6524 [hep-ph].

[13] C. Bogner and F. Brown, Feynman integrals and iterated integrals on moduli spaces of curves of genus zero, Commun. Num. Theor. Phys. 09 (2015) 189-238, arXiv:1408.1862 [hep-th].

[14] C. Bogner and M. Lüders, Multiple polylogarithms and linearly reducible Feynman graphs, to appear in the proceedings of the workshop "Periods and Motives", Madrid, July 2012, arXiv:1302.6215 [hep-ph].

[15] C. Bogner and S. Weinzierl, Periods and Feynman integrals, J. Math. Phys. 50, 042302 (2009), arXiv:0711.4863 [hep-th].

[16] C. Bogner and S. Weinzierl, Feynman Graph Polynomials, Int.J.Mod.Phys. A25 (2010) 25852618, arXiv:1002.3458 [hep-ph].

[17] F. Brown, Multiple zeta values and periods of moduli spaces $M_{0, n}$, Ann. Sci. Ec. Norm. Sup'er. (4) 42 (2009), 371-489, [math.AG/0606419].

[18] F. Brown, The massless higher-loop two-point function, Commun. Math. Phys. 287(3):925958, (2009).

[19] F. Brown, On the periods of some Feynman integrals, 2009, math.AG/0910.0114.

[20] F. Brown, Iterated integrals in quantum field theory, in A. Cardona, I. Contreras and A.F. Reyes-Lega, editors, Geometric and Topological Methods for Quantum Field Theory, chapter 5, pages 188-240, Cambridge University Press, 2013.

[21] F. Brown, On the decomposition of motivic multiple zeta values, arXiv:1102.1310 [math.NT].

[22] F. Brown, Irrationality proofs for zeta values, moduli spaces and dinner parties, arXiv:1412.6508[math.NT].

[23] F. Brown and D. Doryn, Framings for graph hypersurfaces, arXiv:1301.3056 [math.AG].

[24] F. Brown and D. Kreimer, Angles, Scales and Parametric Renormalization, Lett.Math.Phys. 103 (2013) 933-1007, arXiv:1112.1180 [hep-th].

[25] F. Brown and O. Schnetz, $A K_{3}$ in $\phi^{4}$, Duke Math. J. Volume 161, Number 10 (2012), 18171862, arXiv:1006.4064v5 [math.AG].

[26] F. Chavez and C. Duhr, Three-mass triangle integrals and single-valued polylogarithms, JHEP 1211 (2012) 114, arXiv:1209.2722 [hep-ph].

[27] K.T. Chen, Iterated path integrals, Bull. Amer. Math. Soc. 83, (1977), 831-879. 
[28] H. Cheng and T.T. Wu, Expanding Protons: Scattering at High Energies, MIT Press, Cambridge, MA, 1987.

[29] L.J. Dixon, J.M. Drummond, C. Duhr and J. Pennington, The four-loop remainder function and multi-Regge behavior at NNLLA in planar $N=4$ super-Yang-Mills theory, JHEP 1406 (2014) 116, arXiv:1402.3300 [hep-th].

[30] L.J. Dixon, J.M. Drummond, M. von Hippel and J. Pennington, Hexagon functions and the three-loop remainder function, JHEP 1312 (2013) 049, arXiv:1308.2276 [hep-th].

[31] J.M. Drummond, G. Papathanasiou and M. Spradlin, A Symbol of Uniqueness: The Cluster Bootstrap for the 3-Loop MHV Heptagon, JHEP 1503 (2015) 072, arXiv:1412.3763 [hep-th].

[32] C. Duhr, Mathematical aspects of scattering amplitudes, arXiv:1411.7538v1 [hep-ph].

[33] C. Duhr, H. Gangl and J. Rhodes, From polygons and symbols to polylogarithmic functions, JHEP 1210 (2012) 075, math-ph/1110.0458.

[34] T. Gehrmann and E. Remiddi, Two loop master integrals for gamma* $\rightarrow 3$ jets: The Planar topologies, Nucl. Phys. B601 (2001) 248-286, arXiv:hep-ph/0008287.

[35] T. Gehrmann and E. Remiddi, Numerical evaluation of harmonic polylogarithms, Comput. Phys. Commun. 141 (2001) 296-312, arXiv:hep-ph/0107173.

[36] T. Gehrmann and E. Remiddi, Numerical evaluation of two-dimensional harmonic polylogarithms, Comput. Phys. Commun. 144 (2002) 200-223, arXiv:hep-ph/0111255.

[37] A.B. Goncharov, Multiple polylogarithms, cyclotomy and modular complexes, Math Res. Letters 5, (1998) 497-516, arXiv:1105.2076 [math.AG].

[38] A.B. Goncharov, A simple construction of Grassmannian polylogarithms, Adv. Math. 241 (2013), 79-102, math.AG/0908.2238v2.

[39] A.B. Goncharov and Y.I. Manin, Multiple $\zeta$-motives and moduli spaces $\overline{\mathcal{M}}_{0, n}$, Compositio Math. 140 (2004), 1-14.

[40] A.B. Goncharov, M. Spradlin, C. Vergu and A. Volovich, Classical Polylogarithms for Amplitudes and Wilson Loops, Phys. Rev. Lett. 105:151605,2010, [hep-th/1006.5703].

[41] T. Hahn and P. Lang, FeynEdit - a tool for drawing Feynman diagrams, Comput. Phys. Commun.179:931-935 (2008), arXiv:0711.1345 [hep-ph].

[42] J. Harris and J. Morrison, Moduli of Curves, Graduate Texts in Mathematics 187, Springer, 1998.

[43] J.M. Henn, A.V. Smirnov and V.A. Smirnov, Evaluating single-scale and/or non-planar diagrams by differential equations, JHEP 1403 (2014) 088, arXiv:1312.2588 [hep-th].

[44] M. Kontsevich and D. Zagier, Periods, in: B. Engquis and W. Schmid, editors, Mathematics unlimited - 2001 and beyond, 771-808, Springer (2001).

[45] A.V. Kotikov, Differential equantions method: New technique for massive Feynman diagrams calculation, Phys. Lett. B254 (1991), 158-164. 
[46] J. A. Lappo-Danilevsky, Théorie algorithmique des corps de Riemann, Rec. Math. Moscou 34 (1927), no. 6 pp. 113-146.

[47] J. A. Lappo-Danilevsky, Mémoires sur la théorie des systèmes des équations différen- tielles linéaires, vol. I-III. Chelsea, 1953.

[48] D. Maitre, HPL, a mathematica implementation of the harmonic polylogarithms, Comput. Phys. Commun. 174 (2006) 222 [hep-ph/0507152].

[49] D. Maitre, Extension of HPL to complex arguments, Comput. Phys. Commun. 183 (2012) 846 [hep-ph/0703052].

[50] A. von Manteuffel, E. Panzer and R.M. Schabinger, A quasi-finite basis for multi-loop Feynman integrals, JHEP 1502 (2015) 120, arXiv:1411.7392 [hep-ph].

[51] D. Mumford, Curves and their Jacobians, appendix to The Red Book of Varieties and Schemes, Second Expanded Edition, Lecture Notes in Mathematics 1358, Springer, 1999.

[52] E. Panzer, On hyperlogarithms and Feynman integrals with divergences and many scales, JHEP 1403 (2014) 071, arXiv:1401.4361 [hep-th].

[53] E. Panzer, Feynman integrals and hyperlogarithms, PhD thesis, Humboldt University, arXiv:1506.07243 [math-ph].

[54] E. Panzer, Algorithms for the symbolic integration of hyperlogarithms with applications to Feynman integrals, Comput. Phys. Commun. 188 (2014) 148-166, arXiv:1403.3385 [hep-th].

[55] E. Panzer and O. Schnetz, The Galois Coaction on $\phi^{4}$ Periods, in preparation.

[56] H. Poincaré, Sur les groupes des équations linéaires, Acta Mathematica 4 (1884), no. 1 pp. 201-312.

[57] E. Remiddi, Differential Equations for Feynman Graph Amplitudes, Nuovo Cim. A110 (1997), 1435. Nucl. Phys. B580 (2000), 485.

[58] E. Remiddi and J.A.M. Vermaseren, Harmonic Polylogarithms, Int. J. Mod. Phys. A15 (2000) 725-754, [hep-ph/9905237].

[59] O. Schnetz, Quantum periods: A census of $\phi^{4}$-transcendentals, Commun.Num.Theor.Phys.4:1-48, 2010, arXiv:0801.2856v2 [hep-th].

[60] J.A.M. Vermaseren, New features of FORM, arXiv:math-ph/0010025.

[61] J. Vollinga and S. Weinzierl, Numerical evaluation of multiple polylogarithms, Comput. Phys. Commun. 167 (2005) 177, hep-ph/0410259 